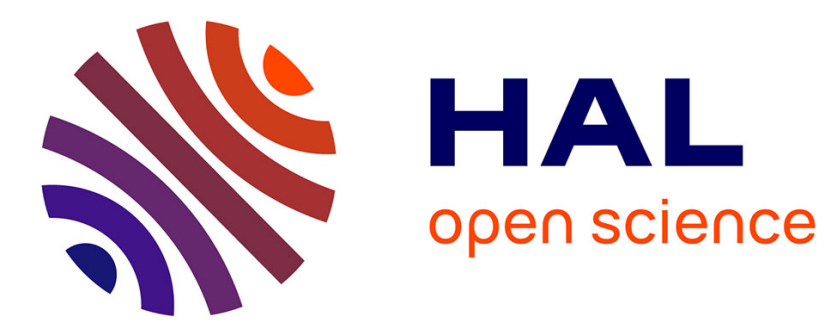

\title{
Boolean universes above Boolean models
}

Friedrich Wehrung

\section{To cite this version:}

Friedrich Wehrung. Boolean universes above Boolean models. The Journal of Symbolic Logic, 1993, 58, no. 4, pp.1219-1250. hal-00004693

\section{HAL Id: hal-00004693 \\ https://hal.science/hal-00004693}

Submitted on 13 Apr 2005

HAL is a multi-disciplinary open access archive for the deposit and dissemination of scientific research documents, whether they are published or not. The documents may come from teaching and research institutions in France or abroad, or from public or private research centers.
L'archive ouverte pluridisciplinaire HAL, est destinée au dépôt et à la diffusion de documents scientifiques de niveau recherche, publiés ou non, émanant des établissements d'enseignement et de recherche français ou étrangers, des laboratoires publics ou privés. 


\title{
Boolean universes above Boolean models
}

\author{
Friedrich WEHRUNG \\ Université de Caen \\ Département de Mathématiques \\ 14032 CAEN CEDEX
}

\begin{abstract}
.
We establish several first- or second-order properties of models of first-order theories by considering their elements as atoms of a new universe of set theory, and by extending naturally any structure of Boolean model on the atoms to the whole universe. For example, complete $f$-rings are "boundedly algebraically compact" in the language $(+,-, \cdot, \wedge, \vee, \leq)$, and the positive cone of a complete $\ell$-group with infinity adjoined is algebraically compact in the language $(+, \vee, \leq)$. We also give an example with any first-order language. The proofs can be translated into "naive set theory" in a uniform way.
\end{abstract}

\section{$\S 0$. Introduction.}

This paper proposes to establish new properties of some known first-order structures, as e.g. lattice-ordered groups but also structures without any ordering, via the study of an associated Boolean model. T. Jech proposes such a method in his paper [13], where he associates to every partially ordered set $E$ a certain definition of forcing, depending only on the ordering of $E$; this forcing yields naturally a Boolean-valued model, which roughly speaking describes the "projections" of $E$. When $E$ is what he calls "Boolean-linear", then these "projections" are linearly ordered and the properties of $E$ can be retrieved from the properties of the Boolean model.

Here, we will also consider forcing over models of arbitrary first-order languages (in particular not necessarily containing a binary relation symbol) and thus there will be several definitions of forcing. This can be done for any language. A typical example of this situation is shown in $\S 3$, where we start with a model $K$ for an arbitrary first-order language L which in addition carries a Hausdorff compact topological structure for which the interpretations of the relations are closed and the interpretations of the functions are continuous, and a complete Boolean algebra $B$. Then there is a canonical way to define the space of $K$-valued $B$-measurable functions, which is to define it as the space $\mathcal{C}(S, K)$ of all continuous functions from the Stone space $S$ of $B$ to $K$. We show in $\S 3$ that elements of this space can be interpreted as the $B$-valued elements of the closure of $K$ in the $B$-valued universe (theorem 3.10), in a very tight way (lemma 3.9). This allows in some sense to argue "componentwise" on elements of $\mathcal{C}(S, K)$ where it would not be possible to do so in normal conditions; as an application, we show that $\mathcal{C}(S, K)$ is algebraically compact in a strong sense (corollary 3.11). There are many first-order structures that are algebraically compact but not topologically compact, for example $\mathbb{Q} / \mathbb{Z}$ in the case of abelian groups [6],

1991 Mathematics subject classification: 03C90, 08A45, 06F05, 06F20, 54H99.

Key words and phrases: Atoms, Boolean models, first-order languages, convergence in lattice-ordered rings, equational compactness, algebraic compactness. 
or the commutative monoid of all Lebesgue-measurable functions from the unit interval to $\overline{\mathbb{N}}=\mathbb{N} \cup\{\infty\}$ modulo null sets (see example 3.1). In fact, algebraic compactness of a structure seems to capture the algebraic information contained in its (virtual) topological compactness.

In $\S 4$, we define a natural forcing over any commutative $\ell$-group, which in addition preserves multiplication if it is equipped with a structure of $f$-ring. This forcing is not limited to positive elements, and is not always equivalent to Jech's forcing, although the Boolean models involved also think they are linearly ordered; however, the results it yields, here about convergence of polynomials in $f$-rings, are valid in the general case.

In $§ 5$, we study again algebraic compactness properties of some first-order structures, this time ordered. One of the main results (theorem 5.5) shows that complete $f$-rings are "boundedly algebraically compact" (in the language $(+,-, \cdot \wedge, \vee, \leq)$ ), i.e. they are algebraically compact for those systems "with bounded solutions". The corollary we obtain (corollary 5.6) solves a problem in [15] about common extensions of charges with values in ordered abelian groups. In an attempt to extend these results to the complete P.O.M.'s introduced in [17], we prove that many complete P.O.M.'s are algebraically compact (theorem 5.9) - actually, one can prove that the P.O.M.'s of theorem 5.9 are exactly the Boolean-linear complete P.O.M.'s (in general, completeness in [13] and in [17] are related, but not equivalent). Conversely, for antisymmetric refinement P.O.M.'s [17], algebraic compactness implies completeness (proposition 5.8).

The set-theoretical context we use here is slightly unorthodox but not really new (see [1], [10, chapter 4]). Instead of embedding our Boolean models into the Scott-Solovay universe $\mathbf{V}^{B}$, which is e.g. done in [13, chapter 9], where it is proved in $Z F C$ that every Boolean-linear space admits a unique completion up to isomorphism (it is really appearent in the "existence" part), we consider the elements of the model that we intend to study as atoms of a universe of set theory (this time $Z F A$ ), and we extend the Boolean model structure of the set of atoms to the whole universe $(\S 2)$, extending the classical construction of $\mathbf{V}^{B}$. This is formalized in $\S 1$ and $\S 2$. While the understanding of $\S 1$ requires a rather rudimentary knowledge of set theory, the prerequisites for $\S 2$ cover roughly speaking the basic knowledge about forcing, the Scott-Solovay universe $\mathbf{V}^{B}$ and Boolean models, see [11], [12]. In $Z F C$, the method without atoms gives about the same results and the slight preliminary complications brought by the viewpoint of atoms may seem at the first sight cumbersome. In fact, this viewpoint has indeed some technical advantages: many isomorphisms in the case without atoms become identities in the case with atoms (in particular in the case of products of models), making most notations much lighter; this is because the choice of our new universe $\mathbf{W}$ with atoms is well-adapted to the model we intend to study. Also, the method with atoms can be generalized to other set theories, and even to "naive set theory"; this is done (informally) in the Afterword; the method without atoms seems to fail to give such a generalization.

We shall use throughout this paper standard set-theoretical notation. For example, $\omega$ will denote the sup of finite ordinals and for every function $f$ defined on a set $X, f[X]$ will denote the direct image of $X$ under $f$, and for any set $Y, f^{-1}[Y]$ will denote the inverse image of $Y$ under $f$. For all sets $X$ and $Y,{ }^{X} Y$ denotes the set of all maps from $X$ to $Y$. If $\varphi(n)$ is any formula (with parameters) the free variable of which has to be 
thought in some directed set $\mathrm{D}$ (given by the context), then $\left(\forall^{\infty} n\right) \varphi(n)$ is the statement $(\exists m \in \mathcal{D})(\forall n \in \mathcal{D})(n \geq m \Rightarrow \varphi(n))$. If $X$ and $Y$ are any subsets of a partially ordered set, $X \leq Y$ is the statement $(\forall x \in X)(\forall y \in Y)(x \leq y)$. If $X=\{a\}$, then we will write $a \leq Y$ instead of $\{a\} \leq Y$, etc.. If $Z$ is a subset of some topological space $X$, we will denote by $\mathrm{Cl}(Z)$ its closure in $X$.

\section{$\S 1$. The universe $\mathbf{V}_{A}$.}

We shall use throughout this paper the context of set theory with atoms - see e.g. [10, chapter 4]. The language consists on $=, \in$ and two constant symbols 0 and $\mathbf{A}$. For convenience of the reader, we list here the axioms of $Z F A$ :

0. Empty set. $\neg \exists x(x \in 0)$.

1. Atoms. $(\forall z)(z \in \mathbf{A} \Leftrightarrow(z \neq 0$ and $\neg(\exists x)(x \in z))$.

The elements of $\mathbf{A}$ will be called atoms; we will sometimes write $\operatorname{set}(x)$ instead of $x \notin \mathbf{A}$, and of course $\emptyset$ instead of 0 .

2. Extensionality. $(\forall \operatorname{set} X, Y)((\forall z)(z \in X \Leftrightarrow z \in Y) \Rightarrow X=Y)$.

3. Regularity. $(\forall$ set $S \neq 0)(\exists x \in S)(\forall y \in S) \neg(y \in x)$.

4. Pairing. $(\forall x, y)(\exists z)(\forall t)(t \in z \Leftrightarrow(t=x$ or $t=y))$.

5. Union. $(\forall X)(\exists$ set $Y)(\forall y)(y \in Y \Leftrightarrow(\exists z \in X)(y \in z))$.

We abbreviate from now on $(\operatorname{set}(X)$ and $\operatorname{set}(Y)$ and $(\forall z \in X)(z \in Y))$ by $X \subseteq Y$.

6. Power Set. $(\forall$ set $X)(\exists$ set $Y)(\forall Z)(Z \in Y \Leftrightarrow Z \subseteq X)$.

7. Separation scheme. $(\forall$ set $X)(\exists$ set $Y)(\forall x)(x \in Y \Leftrightarrow(x \in X$ and $\varphi(x)))$, where $\varphi$ is any formula (with parameters).

8. Collection scheme. $(\forall$ set $X)(\exists$ set $Y)(\forall x \in X)((\exists y) \varphi(x, y) \Rightarrow(\exists y \in Y) \varphi(x, y))$, where $\varphi$ is any formula (with parameters).

9. Axiom of infinity. ( $\exists$ set $X)(0 \in X$ and $(\forall x \in X)(x \cup\{x\} \in X))$.

Let $A$ be any set in a universe $\mathfrak{U}$ of set theory (e.g. $Z F$, or $Z F$ minus the axiom of regularity, or $Z F A$, etc.). We shall construct within $\mathfrak{U}$ a canonical universe $\left(\mathbf{V}_{A}, \in_{A}\right)$ of $Z F A$ with set of atoms [isomorphic to] $A$. First, we define an increasing $O N$-sequence of sets in $\mathfrak{U}$ as follows:

$$
\begin{cases}\mathbf{V}_{A, 0}=\emptyset ; & \\ \mathbf{V}_{A, \xi+1}=(A \times\{0\}) \cup\left(\mathcal{P}\left(\mathbf{V}_{A, \xi}\right) \times\{1\}\right) & (\text { all } \xi \text { in } O N) \\ \mathbf{V}_{A, \lambda}=\bigcup_{\xi<\lambda} \mathbf{V}_{A, \xi} & (\text { all limit } \lambda \text { in } O N)\end{cases}
$$

and then $\mathbf{V}_{A}=\bigcup_{\xi \in O N} \mathbf{V}_{A, \xi}$. Furthermore, $\epsilon_{A}$ is naturally defined on $\mathbf{V}_{A}$ by

$$
x \in_{A} y \Leftrightarrow(\exists \bar{y})(y=(\bar{y}, 1) \text { and } x \in \bar{y}) .
$$

We will of course identify in the notation $\mathbf{V}_{A}$ and $\left(\mathbf{V}_{A}, \in_{A}\right)$. Then, the proof of the following proposition is an easy exercise:

1.1. Proposition. $\mathbf{V}_{A}$ is a model of $Z F A$, with 0 interpreted by $\tilde{0}=(\emptyset, 1)$ and $\mathbf{A}$ by $\tilde{A}=(A \times\{0\}, 1)$, so that the atoms of $\mathbf{V}_{A}$ are the elements of the form $(a, 0), a \in A$. Furthermore, if $\mathfrak{U}$ satisfies the axiom of choice, then $\mathbf{V}_{A}$ satisfies the axiom of choice. 
The fact that $\mathbf{V}_{A}$ inherits the axiom of choice from $\mathfrak{U}$ also holds for the countable axiom of choice, the Boolean prime ideal theorem, the Hahn-Banach extension theorem, etc.. The proofs are easy exercises, some of them using the coming proposition 1.2.

The main feature of $\mathbf{V}_{A}$ is that if $A$ is equipped with some additional structure (group, topological space, etc.), then $\mathbf{V}_{A}$ captures all the "mathematical" information about this structure (after identification of every $a$ in $A$ with $(a, 0)$ ); in particular, $A \cong B$ implies $\mathbf{V}_{A} \cong \mathbf{V}_{B}$. For example, if $R$ is any subset of $A$, then $R$ is coded in $\mathbf{V}_{A}$ by $\tilde{R}=(R \times\{0\}, 1)$, etc.. More generally, if $A$ carries a higher order structure (e.g. topological space), then $\tilde{A}$ carries in $\mathbf{V}_{A}$ an isomorphic (in a natural sense) structure. We can e.g. make this more precise in the following way. If $\alpha$ is an ordinal and $S$ is a set, define inductively $\mathcal{P}^{\alpha}(S)$ by $\mathcal{P}^{0}(S)=S, \mathcal{P}^{\alpha+1}(S)=\mathcal{P}\left(\mathcal{P}^{\alpha}(S)\right)$ and $\mathcal{P}^{\lambda}(S)=\prod_{\alpha<\lambda} \mathcal{P}^{\alpha}(S)$ for limit $\lambda$; furthermore, denote by $\mathbf{V}^{\mathfrak{U}}$ the Von Neumann universe of $\mathfrak{U}$ (i.e. those sets in $\mathfrak{U}$ for which the rank is defined). Then, it is easy to prove the following proposition:

\subsection{Proposition.}

(i) $\varphi: \mathbf{V}^{\mathfrak{U}} \rightarrow \mathbf{V}^{\mathbf{V}_{A}}$ defined inductively by $\varphi(x)=(\varphi[x], 1)$ is an isomorphism.

(ii) For every ordinal $\alpha$ of $\mathfrak{U}$, one can define by induction on $\alpha$ an isomorphism $\varphi_{\alpha}$ from $\mathcal{P}^{\alpha}(A)$ (in $\mathfrak{U}$ ) onto $\mathcal{P}^{\varphi(\alpha)}(\tilde{A})$ (in $\mathbf{V}_{A}$ ) by $\varphi_{0}(a)=(a, 0) ; \varphi_{\alpha+1}(x)=\left\{\varphi_{\alpha}(y): y \in x\right\}$; and $\varphi_{\lambda}\left(\left\langle x_{\alpha}: \alpha<\lambda\right\rangle\right)=\left\langle\varphi_{\alpha}\left(x_{\alpha}\right): \alpha<\lambda\right\rangle$ for limit $\lambda$.

As a consequence of (i), one can say that $\mathfrak{U}$ and $\mathbf{V}_{A}$ have the same "basic mathematical objects", as e.g. $\mathbb{N}, \mathbb{R}$, etc. . As a consequence of proposition 1.2, to prove a certain property about a given mathematical structure with $A$ as underlying set, one may work without loss of generality "as if the elements of $A$ were atoms of the universe".

\section{$\S 2$. Boolean-valued universes with atoms.}

We refer the reader to [12] for the general definition and basic properties of Boolean models. Note that we will not require our Boolean models to satisfy the axiom $(\forall x, y)(\|x=y\|=1 \Rightarrow x=y)$.

Suppose now that we are given a universe $\mathbf{W}$ of $Z F A$, with set of atoms $\mathbf{A}$ (possibly empty). Let $B$ be a complete Boolean algebra of $\mathbf{W}$. Define inductively $\mathbf{W}_{\alpha}^{B}(\alpha \in O N)$ the following way:

$$
\begin{cases}\mathbf{W}_{0}^{B}=\emptyset & (\text { all } \alpha \in O N) \\ \mathbf{W}_{\alpha+1}^{B}=\mathbf{A} \cup \bigcup_{X \subseteq \mathbf{W}_{\alpha}^{B}}{ }^{X} B & (\text { all limit } \lambda \in O N) \\ \mathbf{W}_{\lambda}^{B}=\bigcup_{\alpha<\lambda} \mathbf{W}_{\alpha}^{B} & \text { (a) }\end{cases}
$$

and then define $\mathbf{W}^{B}=\bigcup_{\alpha \in O N} \mathbf{W}_{\alpha}^{B}$.

Now, we make the following assumption:

$\mathbf{A}$ is equipped with a structure of $B$-valued model of the equality (i.e. a map $\mathbf{A} \times \mathbf{A} \rightarrow B$, $(a, b) \mapsto \llbracket a=b \rrbracket$ such that for all $a, b, c$ in $\mathbf{A}, \llbracket a=a \rrbracket=1, \llbracket a=b \rrbracket=\llbracket b=a \rrbracket$, $\llbracket a=b \rrbracket \wedge \llbracket b=c \rrbracket \leq \llbracket a=c \rrbracket)$. 
Then, for all $\boldsymbol{x}, \boldsymbol{y}$ in $\mathbf{W}^{B}$, define inductively the following Boolean values:

$$
\left\{\begin{array}{l}
\|\boldsymbol{x} \in \boldsymbol{y}\|=\bigvee_{\boldsymbol{z} \in \operatorname{dom}(\boldsymbol{y})}(\|\boldsymbol{x}=\boldsymbol{z}\| \wedge \boldsymbol{y}(\boldsymbol{z})) \\
\left\|\boldsymbol{x} \subseteq^{*} \boldsymbol{y}\right\|=\bigwedge_{\boldsymbol{z} \in \operatorname{dom}(\boldsymbol{x})}(\boldsymbol{x}(\boldsymbol{z}) \rightarrow\|\boldsymbol{z} \in \boldsymbol{y}\|) \\
\|\boldsymbol{x}=\boldsymbol{y}\|= \begin{cases}\llbracket \boldsymbol{x}=\boldsymbol{y} \rrbracket & (\boldsymbol{x}, \boldsymbol{y} \in \mathbf{A}) \\
0 & (\boldsymbol{x} \in \mathbf{A} \text { and } \boldsymbol{y} \notin \mathbf{A}, \text { or } \boldsymbol{x} \notin \mathbf{A} \text { and } \boldsymbol{y} \in \mathbf{A}) \\
\left\|\boldsymbol{x} \subseteq^{*} \boldsymbol{y}\right\| \wedge\left\|\boldsymbol{y} \subseteq^{*} \boldsymbol{x}\right\| & (\boldsymbol{x}, \boldsymbol{y} \notin \mathbf{A})\end{cases}
\end{array}\right.
$$

Furthermore, for all $x$ in $\mathbf{W}$, define inductively $\check{x}$ by

$$
\check{a}=a(\text { all } a \in \mathbf{A}) \text { and } \check{x}=\{(\check{y}, 1): y \in x\} \text { for all } x \in \mathbf{W} \backslash \mathbf{A} \text {; }
$$

then, for all $\boldsymbol{x}$ in $\mathbf{W}^{B}$, define $\|\underline{\mathbf{W}} \boldsymbol{x}\|=\bigvee_{y \in \mathbf{W}}\|\boldsymbol{x}=\check{y}\|$. Here, $\underline{\mathbf{W}}$ will be a unary relational symbol.

Then, the basic features of these objects look very much like the ones of the classical Scott-Solovay universe $\mathbf{V}^{B}$ ( see [11, chapter 18]). For instance, the definition (2.2) is indeed well-founded. Furthermore, it defines a $B$-valued model:

2.1. Lemma. $\mathbf{W}^{B}$, equipped with $\left\|_{-}\right\|$defined above, is a $B$-valued model of the language $(=, \in, \underline{\mathbf{W}})$.

This allows us to define the usual way (see [11, chapter 18]) the Boolean value of any statement of $(=, \in, \underline{\mathbf{W}})$ with parameters from $\mathbf{W}^{B}$, so that the Boolean value of any tautological statement is equal to 1 . Note that we have in particular

$$
\left\|\boldsymbol{x} \subseteq^{*} \boldsymbol{y}\right\|=\|(\forall \boldsymbol{z})(\boldsymbol{z} \in \boldsymbol{x} \Rightarrow \boldsymbol{z} \in \boldsymbol{y})\|
$$

for all $\boldsymbol{x}, \boldsymbol{y}$ in $\mathbf{W}^{B}$.

Let $Z F A(\underline{\mathbf{W}})$ be the theory $Z F A$ in which the separation scheme and the collection scheme are written for all formulas of $(=, \in, \underline{\mathbf{W}})$. Then, as for the case $\mathbf{A}=\emptyset$, one can prove the following

2.2. Lemma. $\mathbf{W}^{B}$ is a $B$-valued model of $Z F A(\underline{\mathbf{W}})$, i.e. for every axiom $\theta$ of $Z F A(\underline{\mathbf{W}})$, we have $\|\theta\|=1$. Furthermore, if $\mathbf{W}$ satisfies the axiom of choice $A C$, then $\|A C\|=1$.

We also have the analogue of fullness of $\mathbf{V}^{B}$ :

2.3. Lemma. $\mathbf{W}^{B} \backslash \mathbf{A}$ is full, i.e. for every antichain $W$ of $B$ and every family $\left(\boldsymbol{x}_{u}\right)_{u \in W}$ of elements of $\mathbf{W}^{B} \backslash \mathbf{A}$, there exists an element $\boldsymbol{x}$ of $\mathbf{W}^{B} \backslash \mathbf{A}$ such that

$$
(\forall u \in W)\left(u \leq\left\|\boldsymbol{x}=\boldsymbol{x}_{u}\right\|\right) \text {; }
$$

Thus, if $\mathbf{W}$ satisfies $A C$, then for every formula $\varphi(x)$ with parameters from $\mathbf{W}^{B}$, there exists $\boldsymbol{a}$ in $\mathbf{W}^{B} \backslash \mathbf{A}$ such that

$$
\|(\exists \text { set } \boldsymbol{x}) \varphi(\boldsymbol{x})\|=\| \varphi(\boldsymbol{a}) \| .
$$


The proofs of lemmas 2.1 to 2.3 can be easily obtained from the usual case without atoms, thus we will not show them here. We refer the reader to [11, chapter 18] for more details.

Until now, the theory looks much like the usual well-known theory without atoms. Is it really the same everywhere? The answer turns out to be no, as a major difference, which will be very important for our study, will be that in the case without atoms, the "sections" of $\mathbf{V}^{B}$ (quotients of $\mathbf{V}^{B}$ by a given generic ultrafilter) correspond to generic extensions of the universe $\mathbf{V}$; while in the general case with atoms, it is no longer the case: indeed, heuristically, if $G$ is a generic ultrafilter on $B$, then $\mathbf{W}^{B} / G$ has $\mathbf{A} / G$ rather than A as set of atoms.

This remark is connected to the fact that $\Delta_{0}$ statements with parameters of the form $\check{x}$ are not necessarily absolute. For example, one may have atoms $a$ and $b$ such that $a \neq b$ but $\|a=b\|=1$; or an atom $a$ and a subset $X$ of $A$ such that $a \notin X$ but $\|a \in \check{X}\|=1$. This leads us to the following

2.4. Definition. A formula $\varphi\left(x_{1}, \ldots, x_{n}\right)$ is $B$-uniform when for all $x_{1}, \ldots, x_{n}$ in $\mathbf{W}$, $\mathbf{W} \models \varphi\left(x_{1}, \ldots, x_{n}\right)$ implies $\left\|\varphi\left(\check{x}_{1}, \ldots, \check{x}_{n}\right)\right\|=1$; a formula is uniform when it is $B$-uniform in every universe of $Z F A$ and for all $B$.

Heuristically, a formula is $B$-uniform when its global satisfaction implies its local satisfaction.

2.5. We will now give a "working list" of uniform or non-uniform formulas (the adaptations of these remarks to the $B$-uniform case are obvious).

(1) Any $\Sigma_{1}^{\mathbf{V}}$-formula, i.e. any relativization to $\mathbf{V}$ of a $\Sigma_{1}$-formula, is uniform.

(2) $\operatorname{set}(x)$ and $x \in \mathbf{A}$ are uniform formulas.

(3) $x \in y, x \subseteq y, x=y$ are uniform.

(4) If $\varphi$ and $\psi$ are uniform, then $\varphi \wedge \psi, \varphi \vee \psi,(\forall x \in y) \varphi$ and $(\exists x) \varphi$ are uniform. Warning: $\neg \varphi$ may not be a uniform formula.

(5) The substitution of a uniform function (i.e. a uniform formula defining a function provably in $Z F A$ ) in a uniform formula is [equivalent to] a uniform formula.

(6) The following formulas are uniform: $z=\{x, y\} ; z=(x, y) ; y=\bigcup x$; " $x$ is an ordered pair"; " $x$ is a relation" (i.e. a set of ordered pairs); " $x$ is a relation and $y=\operatorname{dom}(x) " ; " x$ is a relation and $y=\operatorname{rng}(x) " ; z=x\lceil y(x\lceil y$ denotes as usual the restriction of $x$ to $y$ ).

(7) $z=x \cap y$, " $x$ is a function" are not uniform statements. If $f$ is a function in $\mathbf{W}$, say that $f$ is $B$-uniform when $\| \check{f}$ is a function $\|=1$. Putting $X=\operatorname{dom}(f)$, this is equivalent to the statement

$$
(\forall x, y \in X)\left(\|\check{x}=\check{y}\| \leq\left\|f(x)^{`}=f(y)^{\smile}\right\|\right) .
$$

Thus this is in particular the case when for all $x, y$ in $X, x \neq y$ implies $\|\check{x}=\check{y}\|=0$; we will say that $X$ is $B$-scattered. For example, elements of $\mathbf{V}$ are always $B$-scattered. 
It turns out that the obstruction to $B$-uniformity of $\Sigma_{1}$-statements is exactly the lack of scatterdness of the set of atoms.

To make this precise, define the content $\operatorname{CONT}(x)$ of any element $x$ of $\mathbf{W}$ to be the set of atoms that are used in the building up of $x$ starting from $\emptyset$ and the atoms: formally, $\operatorname{CONT}(a)=\{a\}$ for all $a$ in $\mathbf{A}$, and, for every set $x, \operatorname{CONT}(x)=\bigcup\{\operatorname{CONT}(y): y \in x\}$. Then we have the following proposition, whose proof is similar to the classical proof without atoms (see [11], lemma 18.11):

2.6. Proposition. Let $\varphi$ be a $\Delta_{0}$-statement with $k$ free variables of the language $(=, \in, \underline{\mathbf{W}})$, let $a_{1}, \ldots, a_{k}$ in $\mathbf{W}$ such that $\bigcup_{1 \leq i \leq k} \operatorname{CONT}\left(a_{i}\right)$ is $B$-scattered. Then we have

$$
\left\|\varphi\left(\check{a}_{1}, \ldots, \check{a}_{k}\right)\right\|= \begin{cases}1 & \left(\mathbf{W} \models \varphi\left(a_{1}, \ldots, a_{k}\right)\right) \\ 0 & \left(\mathbf{W} \not \models \varphi\left(a_{1}, \ldots, a_{k}\right)\right)\end{cases}
$$

Thus, if $\mathbf{A}$ is $B$-scattered, then $\Sigma_{1}$-statements are $B$-uniform.

The following corollary will be useful:

2.7. Corollary. For all $X$ in $\mathbf{W}$ and all $\boldsymbol{Y}$ in $\mathbf{W}^{B}$, we have

$$
\| \boldsymbol{Y} \subseteq \check{X} \text { and } \boldsymbol{Y} \text { is finite }\left\|=\bigvee_{Z \text { finite } \subseteq X}\right\| \boldsymbol{Y}=\check{Z} \| .
$$

Proof. By proposition 2.6, $\| \check{\omega}$ is the first limit ordinal $\|=1$. Thus it suffices to prove that for every $n$ in $\omega$ and every $\boldsymbol{f}$ in $\mathbf{W}^{B}$, we have

$$
\| \boldsymbol{f} \text { is a surjection } \check{n} \rightarrow \boldsymbol{Y} \text { and } \boldsymbol{Y} \subseteq \check{X}\left\|\leq \bigvee_{Z \text { finite } \subseteq X}\right\| \boldsymbol{Y}=\check{Z} \| .
$$

Let $u$ be the left-hand side of (2.3), let $v \leq u$ non zero. It suffices to prove that $v$ is not disjoint from the right-hand side of (2.3). But it is easy to construct inductively $v_{i}(i \leq n)$ in $B \backslash\{0\}$ and $x_{i}(i<n)$ in $X$ such that $v_{0}=v$ and for all $i<n, v_{i+1}=v_{i} \wedge\left\|\boldsymbol{f}(\check{l})=\check{x}_{i}\right\|$. Thus $v_{n} \leq\|\boldsymbol{Y}=\check{Z}\|$ where $Z=\left\{x_{i}: i<n\right\}$, which completes the proof.

2.8. Definition. A set $X$ in $\mathbf{W}$ is $B$-projectable when the following holds:

$$
(\forall u \in B)(\forall x, y \in X)(\exists z \in X)(u \leq\|\check{x}=\check{z}\| \text { and } \neg u \leq\|\check{y}=\check{z}\|)
$$

It is easy to see that $B$-projectability of $X$ is equivalent to the stronger property that for every finite antichain $W$ of $B$ and every family $\left(x_{u}\right)_{u \in W}$ of elements of $X$, there exists $x$ in $X$ such that $(\forall u \in W)\left(u \leq\left\|\check{x}=\check{x}_{u}\right\|\right)$. A stronger property is obtained by removing the finiteness assumption on $W$ :

2.9. Definition. A set $X$ is $\mathbf{W}$ is $B$-full when for every antichain $W$ of $B$ and every family $\left(x_{u}\right)_{u \in W}$ of elements of $X$, there exists $x$ in $X$ such that

$$
(\forall u \in W)\left(u \leq\left\|\check{x}=\check{x}_{u}\right\|\right) .
$$


For example, when $B$ is non trivial and there are no atoms, the only $B$-projectable (and $B$-full) sets are $\emptyset$ and the singletons. $\mathbf{W}^{B}$ :

Both projectability and fullness give raise to logical properties of the Boolean universe

2.10. Lemma. Let $X$ be a set in $\mathbf{W}$ which is either finite or $B$-projectable. Then $X$ is weakly $B$-projectable, i.e.

$$
\left(\forall \boldsymbol{Y} \in \mathbf{W}^{B}\right)\left(\|\boldsymbol{Y} \subseteq \check{X}\|=\bigvee_{Z \subseteq X}\|\boldsymbol{Y}=\check{Z}\|\right) .
$$

Proof. By 2.5, the formula $z=x \cup y$ is uniform; it follows easily that any finite union of weakly B-projectable sets is weakly B-projectable. Since singletons are weakly $B$-projectable (the verification is immediate), any finite set is weakly $B$-projectable. Now, let $X$ be a $B$-projectable set, let $\boldsymbol{Y}$ in $\mathbf{W}^{B}$. Put $u=\|\boldsymbol{Y} \subseteq \check{X}\|, v=\bigvee_{Z \subset X}\|\boldsymbol{Y}=\check{Z}\|$. It is immediate that $v \leq u$. Conversely, we have $u=u_{0} \vee u_{1}$ where $u_{0}=\|\boldsymbol{Y}=\emptyset\|$ and $u_{1}=\|\emptyset \neq \boldsymbol{Y} \subseteq \check{X}\| ;$ it is obvious that $u_{0} \leq v$, so it remains to prove that $u_{1} \leq v$. But $u_{1}=\bigvee_{a \in X} v_{a}$ where we put $v_{a}=\|\check{a} \in \boldsymbol{Y} \subseteq \check{X}\|$, so it suffices to prove that $v_{a} \leq v$ for all $a$ in $X$. Let $Z=\left\{x \in X: v_{a} \leq\|\check{x} \in \boldsymbol{Y}\|\right\}$.

Claim. $v_{a} \leq\|\boldsymbol{Y}=\check{Z}\|$.

Proof of claim. Since $\|\operatorname{set}(\boldsymbol{Y})\|=\|\operatorname{set}(\check{Z})\|=1$, it suffices to prove that $v_{a} \leq \| \boldsymbol{Y} \subseteq^{*}$ $\check{Z} \|$ and $v_{a} \leq\left\|\check{Z} \subseteq^{*} \boldsymbol{Y}\right\|$. The second inequality is immediate by definition of $Z$. Conversely, since $v_{a} \leq\|\boldsymbol{Y} \subseteq \check{X}\|$, it suffices to prove that for all $x$ in $X$, we have $v_{a} \leq(\|\check{x} \in \boldsymbol{Y}\| \rightarrow$ $\|\check{x} \in \check{Z}\|)$, i.e. $v_{a} \wedge\|\check{x} \in \boldsymbol{Y}\| \leq\|\check{x} \in \check{Z}\|$. Let $w$ be the left-hand side of this inequality. Since $x \in X, a \in X$ and $w \wedge\left(v_{a} \backslash w\right)=0$, there exists, by $B$-projectability of $X$, some $y$ in $X$ such that $w \leq\|\check{x}=\check{y}\|$ and $v_{a} \backslash w \leq\|\check{a}=\check{y}\|$. Thus $v_{a} \leq\|\check{y} \in \boldsymbol{Y}\|$, whence $y \in Z$. Thus $w \leq\|\check{x} \in \check{Z}\|$, which concludes the proof of the claim.

- Claim .

It follows that $v_{a} \leq v$, which concludes the proof.

It follows immediately that if $\varphi$ is any formula with parameters from $\mathbf{W}^{B}$ and $X$ is any finite or $B$-projectable set, then

$$
\|(\exists \boldsymbol{Y} \subseteq \check{X}) \varphi(\boldsymbol{Y})\|=\bigvee_{Y \subseteq X}\|\varphi(\check{Y})\|
$$

and

$$
\|(\forall \boldsymbol{Y} \subseteq \check{X}) \varphi(\boldsymbol{Y})\|=\bigwedge_{Y \subseteq X}\|\varphi(\check{Y})\|
$$

The logical property resulting from fullness is more classical:

2.11. Lemma. Suppose that $\mathbf{W}$ satisfies $A C$. Let $X$ be a nonempty $B$-full element of $\mathbf{W}$, let $\varphi(x)$ be a formula with parameters from $\mathbf{W}^{B}$. Then there exists $a$ in $X$ such that

$$
\|(\exists \boldsymbol{x} \in \check{X}) \varphi(\boldsymbol{x})\|=\|\varphi(\check{a})\| .
$$

Proof. Similar to the (classical) proof of lemma 2.3. 
The following proposition does not have any non trivial analogue in set theory without atoms:

2.12. Proposition. Let $X$ be a $B$-projectable set. Then $\mathcal{P}(X) \backslash\{\emptyset\}$ is $B$-projectable. Furthermore, if $\mathbf{W}$ satisfies $A C$ and $X$ is $B$-full, then $\mathcal{P}(X) \backslash\{\emptyset\}$ is $B$-full.

Proof. Suppose that $X$ is $B$-projectable. Let $W$ be a finite antichain of $B$, let $\left(X_{u}\right)_{u \in W}$ be a family of nonempty subsets of $X$. Define $Y$ as

$$
Y=\left\{x \in X:(\forall u \in W)\left(u \leq\left\|\check{x} \in \check{X}_{u}\right\|\right)\right\}
$$

Claim. $(\forall u \in W)\left(u \leq\left\|\check{Y}=\check{X}_{u}\right\|\right)$.

Proof of claim. Fix $u$ in $W$. By definition, it is immediate that $u \leq\left\|\check{Y} \subseteq \check{X}_{u}\right\|$. Conversely, let $x$ in $X_{u}$. For all $v$ in $W \backslash\{u\}$, pick $x_{v}$ in $X_{v}$; put $x_{u}=x$. Since $X$ is $B$ - projectable, there exists $y$ in $X$ such that $(\forall v \in W)\left(v \leq\left\|\check{y}=\check{x}_{v}\right\|\right)$. Thus $y \in Y$ by definition, whence $u \leq\|\check{x} \in \check{Y}\|$; thus, $u \leq\left\|\check{X}_{u} \subseteq \check{Y}\right\|$. Since the $X_{u}$ 's and $Y$ are sets, the conclusion of the claim follows.

- Claim .

Thus we can conclude in the projectable case. The proof for fullness is similar.

2.13. Remark. It is easy to see that $\mathcal{P}(\{\emptyset\})=\{\emptyset,\{\emptyset\}\}$ is never $B$-projectable, unless $B$ is trivial.

\section{$\S 3$. Measurable functions with values in a compact Hausdorff space.}

Let us start with a classical example.

3.1. ExAmple. Let $E$ be the space of all Lebesgue-measurable functions from the unit interval $[0,1]$ to $\overline{\mathbb{N}}=\mathbb{N} \cup\{\infty\}$ modulo null sets. If $S$ is the Stone space of the random algebra $B_{\omega}$ (=the complete Boolean algebra of Lebesgue-measurable subsets of $[0,1]$ modulo null sets), then it is well-known that $E$ is canonically isomorphic to the space $\mathcal{C}(S, \overline{\mathbb{N}})$ of all continuous maps from $S$ to $\overline{\mathbb{N}}$.

It is also well-known that elements of $E$ can also be interpreted as $B_{\omega}$-valued elements of $\overline{\mathbb{N}}$ (and that similar results also hold when replacing $\overline{\mathbb{N}}$ by $\overline{\mathbb{P}}$ ). We shall now generalize this fact.

In the general case, let $B$ be a complete Boolean algebra, let $K$ be a compact Hausdorff space. Following the idea of the previous example, the canonical way to define the space of all $B$-measurable $K$-valued functions is to define it as the space $\mathcal{C}(S, K)$ of all continuous functions from the Stone space $S=\operatorname{Ult}(B)$ of $B$ to $K$. Note that $S$ is a compact Hausdorff space, which is in addition extremally disconnected, i.e. the closure of any open set is still open. If $\varphi(s)$ is any formula with one free variable $s$ (and with parameters) such that $X=\{s \in S: \varphi(s)\}$ is open modulo nowhere dense in $S$, we shall denote by $[s: \varphi(s)]$ the unique clopen subset of $S$ equivalent to $X$ modulo the ideal of nowhere dense subsets of $S$, which we will identify with the corresponding element of $B$. Finally, equip $K$ with the set 
$\mathcal{E}$ of all symmetric, closed entourages for the unique uniform structure on $K$ compatible with its topology.

From now on until theorem 3.10, assume that we work in a universe $\mathbf{W}$ of $Z F A$, with all elements of $K$ being atoms; furthermore, we equip $K$ with the unique structure of $B$-scattered structure of $B$-valued model of equality, i.e. $\llbracket a=b \rrbracket=0$ for all distinct $a, b$ in $K$ (see 2.5, (7)).

We will not need the axiom of choice until corollary 3.11 , where it plays an essential role.

For technical reasons, due to the lack of fullness of the set of atoms, we will consider $K^{\bullet}=\{\{x\}: x \in K\}$, equipped with the uniformity basis $\mathcal{E}^{\bullet}$ corresponding to E. Furthermore, for all $a$ in $K$, we will note $a^{\bullet}$ instead of $\{a\}$; for all $n$ in $\omega \backslash\{0\}$ and all $R \subseteq{ }^{n} K$, we will note $R^{\bullet}$ the corresponding subset of ${ }^{n} K^{\bullet}$.

It follows immediately from proposition 2.6 that the following holds:

$\| \check{\mathcal{E}}^{\bullet}$ is a basis of symmetric closed entourages for a precompact

Hausdorff uniform structure on $\check{K}^{\bullet} \|=1$

Therefore, there is a canonical element $\bar{K}$ of $\mathbf{W}^{B}$ such that

$$
\| \bar{K} \text { is the completion of } \check{K}^{\bullet} \text { relatively to } \check{\mathcal{E}}^{\bullet} \|=1 \text {. }
$$

Let $\mathcal{S}(S, K)$ be the set of all elements of $\mathcal{C}(S, K)$ whose range is finite. For all $x$ in $\mathcal{S}(S, K)$, put

$$
\dot{x}=\{(a,[s: x(s)=a]): a \in K\} .
$$

3.2. Lemma. For all $x$ in $\mathcal{S}(S, K)$ and all $a$ in $K$, we have

$$
\left\|\dot{x}=\check{a}^{\bullet}\right\|=[s: x(s)=a] .
$$

Consequently, $\left\|\dot{x} \in \check{K}^{\bullet}\right\|=1$.

Proof. Put $u_{a}=[s: x(s)=a]$. Since $\dot{x}(a)=u$, we have $u_{a} \leq\|a \in \dot{x}\|$. Furthermore, we have $\|\dot{x} \subseteq\{a\}\|=\bigwedge_{b \in K}(\dot{x}(b) \rightarrow\|b=a\|)=\bigwedge_{b \neq a} \neg u_{b}=u_{a}$ (the last equality comes from the fact that $x$ has finite range), whence the conclusion follows.

3.3. Lemma. Let $n$ in $\omega \backslash\{0\}$, let $R \subseteq{ }^{n} K$. Then for all $x_{i}(i<n)$ in $\mathcal{C}(S, K)$, we have

$$
\left\|\left(\dot{x}_{i}\right)_{i<\check{n}} \in \check{R}^{\bullet}\right\|=\left[s:\left(x_{i}(s)\right)_{i<n} \in R\right] .
$$

Proof. A simple calculation:

$$
\begin{aligned}
\left\|\left(\dot{x}_{i}\right)_{i<\check{n}} \in \check{R}^{\bullet}\right\| & =\bigvee_{\left(a_{i}\right)_{i<n} \in R} \bigwedge_{i<n}\left\|\dot{x}_{i}=\check{a}_{i}^{\bullet}\right\|= \\
& =\bigwedge_{\left(a_{i}\right)_{i<n} \in R}\left[s:\left(x_{i}(s)\right)_{i<n}=\left(a_{i}(s)\right)_{i<n}\right]=\left[s:\left(x_{i}(s)\right)_{i<n} \in R\right],
\end{aligned}
$$

the second equality coming from lemma 3.2 and the third from the fact that the map $s \mapsto\left(x_{i}(s)\right)_{i<n}$ has finite range. 
Now, we shall extend the map $x \mapsto \dot{x}$ to the whole $\mathcal{C}(S, K)$. From 3.4 to 3.8 , fix $x$ in $\mathcal{C}(S, K)$. For all $\varepsilon$ in $\mathrm{E}$, put

$$
F_{x \varepsilon}=\{y \in \mathcal{S}(S, K):(\forall s \in S)((x(s), y(s)) \in \varepsilon)\}
$$

Since $K$ is precompact, $F_{x \varepsilon}$ is nonempty. Then, let $\boldsymbol{X}_{x \varepsilon}=\left\{(\dot{y}, 1): y \in F_{x \varepsilon}\right\}$. Thus, $\left\|\boldsymbol{X}_{x \varepsilon} \subseteq \check{K}^{\bullet}\right\|=1$.

3.4. Lemma. Let $\varepsilon$ in $E$; put $\eta=\varepsilon \circ \varepsilon$. Then $\left\|\boldsymbol{X}_{x \varepsilon} \times \boldsymbol{X}_{x \varepsilon} \subseteq \check{\eta}^{\bullet}\right\|=1$.

Proof. A simple calculation, using lemma 3.3:

$$
\begin{aligned}
\left\|\boldsymbol{X}_{x \varepsilon} \times \boldsymbol{X}_{x \varepsilon} \subseteq \check{\eta}^{\bullet}\right\| & =\bigwedge_{y, z \in F_{x \varepsilon}}\left\|(\dot{y}, \dot{z}) \in \check{\eta}^{\bullet}\right\| \\
& =\bigwedge_{y, z \in F_{x, \varepsilon}}[s:(y(s), z(s)) \in \eta] \\
& =1 .
\end{aligned}
$$

Let $\mathcal{F}_{x}$ be the canonical $B$-valued name for the filter on $\check{K}^{\bullet}$ generated by the $\boldsymbol{X}_{x \varepsilon}$ 's, $\varepsilon \in \mathcal{E}$, and let $\overline{\mathcal{F}}_{x}$ be the canonical $B$-valued name for the filter on $\bar{K}$ generated by the closures of the elements of $\mathcal{F}_{x}$.

3.5. Lemma. $\| \overline{\mathcal{F}}_{x}$ is a Cauchy filter of closed subsets of $\bar{K} \|=1$.

Proof. It suffices to prove that $\| \mathcal{F}_{x}$ is a Cauchy filter on $\check{K}^{\bullet} \|=1$. But this results immediately from lemma 3.4.

If $X$ and $Y$ are two subsets from a given uniform space, say that $X$ is uniformly interior to $Y$ when there exists an entourage $\varepsilon$ such that $X \circ \varepsilon \subseteq Y$, where $X \circ \varepsilon=\{y$ : $(\exists x \in X)((y, x) \in \varepsilon)\}$.

3.6. Lemma. Let $\varepsilon$ in $E$. Then

$$
\| \mathrm{Cl}\left(\boldsymbol{X}_{x \varepsilon}\right) \text { is uniformly interior to } \mathrm{Cl}\left(\boldsymbol{X}_{x, \varepsilon \circ \varepsilon}\right) \text { in } \bar{K} \|=1 \text {. }
$$

Proof. Put $\eta=\varepsilon \circ \varepsilon$. Since $\| \check{K}^{\bullet}$ is dense in $\bar{K} \|=1$, it suffices to prove that $\| \boldsymbol{X}_{x \varepsilon}$ is uniformly interior to $\boldsymbol{X}_{x \eta}$ in $\check{K}^{\bullet} \|=1$.

To prove this, we prove that the following Boolean value is equal to 1 :

$$
\left\|\boldsymbol{X}_{x \varepsilon} \circ \check{\varepsilon}^{\bullet} \subseteq \boldsymbol{X}_{x \eta}\right\|=\bigwedge_{\substack{y \in F_{x \varepsilon} \\ a \in K}}\left(\left\|\left(\dot{y}, \check{a}^{\bullet}\right) \in \check{\varepsilon}^{\bullet}\right\| \rightarrow\left\|\check{a}^{\bullet} \in \boldsymbol{X}_{x \eta}\right\|\right) \text {. }
$$

So fix $y$ in $F_{x \varepsilon}$ and $a$ in $K$; put $u=\left\|\left(\dot{y}, \check{a}^{\bullet}\right) \in \check{\varepsilon}^{\bullet}\right\|$. By lemma 3.3, $u=[s:(y(s), a) \in$ $\varepsilon]$. Let $s$ in $u$. Then $(x(s), y(s)) \in \varepsilon$ and $(y(s), a) \in \varepsilon$, whence $(x(s), a) \in \eta$. Using precompactness of $K$, it is then easy to find $z$ in $\mathcal{S}(S, K)$ such that $(\forall s \in u)(z(s)=a)$ and $(\forall s \in \neg u)(x(s), z(s)) \in \eta$. Thus $z \in F_{x \eta}$, whence $u \leq\|\check{a} \bullet=\dot{z}\|$, whence $u \leq\left\|\check{a} \bullet \in \boldsymbol{X}_{x \eta}\right\|$, which completes the proof. 
Thus, we get immediately the

3.7. Lemma. $\| \overline{\mathcal{F}}_{x}$ is the filter of neighborhoods of a unique point of $\bar{K} \|=1$.

Note that in the particular case when $x \in \mathcal{S}(S, K)$, we have $x \in F_{x \varepsilon}$ for all $\varepsilon$, whence

$$
\| \overline{\mathcal{F}}_{x} \text { is the filter of neighborhoods of } \dot{x} \|=1 \text {. }
$$

Thus, it is consistent to denote still by $\dot{x}$ the canonical $B$-valued name for the limit of $\overline{\mathcal{F}}_{x}$ in $\bar{K}$.

We will need one more lemma about the $\boldsymbol{X}_{x \varepsilon}$ 's:

3.8. Lemma. For all $\varepsilon$ in $E$ and all $x$ in $\mathcal{C}(S, K)$, we have

$$
\| \boldsymbol{X}_{x \varepsilon} \text { is a closed subset of } \check{K}^{\bullet} \|=1 \text {. }
$$

Proof. It suffices to prove that for all $a$ in $K$, if $u=\left\|\check{a} \bullet \in \operatorname{Cl}\left(\boldsymbol{X}_{x \varepsilon}\right)\right\|$ and $v=\| \check{a} \bullet \in$ $\boldsymbol{X}_{x \varepsilon} \|$, then $u \leq v$. This is done by an easy sequence of calculations:

$$
\begin{aligned}
u & =\bigwedge_{\eta \in \mathcal{E}} \bigvee_{y \in F_{x \varepsilon}}\left\|\left(\dot{y}, \check{a}^{\bullet}\right) \in \check{\eta}^{\bullet}\right\| \\
& =\bigwedge_{\eta \in \mathcal{E}} \bigvee_{y \in F_{x \varepsilon}}[s:(y(s), a) \in \eta] \quad \text { by lemma } 3.3 \\
& \leq \bigwedge_{\eta \in \mathcal{E}}[s:(x(s), a) \in \eta \circ \varepsilon] \\
& =[s:(x(s), a) \in \mathrm{Cl}(\varepsilon)] \\
& =[s:(x(s), a) \in \varepsilon] \quad \text { because } \varepsilon \text { is closed } \\
& =\bigvee_{y \in F_{x \varepsilon}}[s: y(s)=a] \\
& =v .
\end{aligned}
$$

Our next lemma is the analogue of lemma 3.3 for arbitrary $x$ in $\mathcal{C}(S, K)$. For all $n$ in $\omega \backslash\{0\}$ and all $R \subseteq{ }^{n} K$, denote by $\bar{R}$ the canonical $B$-valued name for the closure of $\check{R}^{\bullet}$ in ${ }^{\check{n}} \bar{K}$.

3.9. Lemma. Let $n$ in $\omega \backslash\{0\}$, let $R$ be a closed subset of ${ }^{n} K$. Then for all $x_{i}(i<n)$ in $\mathcal{C}(S, K)$, we have

$$
\left\|\left(\dot{x}_{i}\right)_{i<\check{n}} \in \bar{R}\right\|=\left[s:\left(x_{i}(s)\right)_{i<n} \in R\right]
$$


Proof. A basic calculation of Boolean values:

$$
\begin{aligned}
\left\|\left(\dot{x}_{i}\right)_{i<\check{n}} \in \bar{R}\right\| & =\bigwedge_{\varepsilon \in \mathcal{E}}\left\|\left(\prod_{i<\check{n}} \operatorname{Cl}\left(\boldsymbol{X}_{x_{i} \varepsilon}\right)\right) \cap \check{R}^{\bullet} \neq \emptyset\right\| \quad \text { by lemma } 3.7 \\
& =\bigwedge_{\varepsilon \in \mathcal{E}}\left\|\left(\prod_{i<\check{n}} \boldsymbol{X}_{x_{i} \varepsilon}\right) \cap \check{R}^{\bullet} \neq \emptyset\right\| \quad \text { by lemma } 3.8 \\
& =\bigwedge_{\varepsilon \in \mathcal{E}} \bigvee\left\{\left\|\left(\dot{y}_{i}\right)_{i<\check{n}} \in \check{R}^{\bullet}\right\|:\left(y_{i}\right)_{i<n} \in \prod_{i<n} F_{x_{i} \varepsilon}\right\}
\end{aligned}
$$

But now, for all $\left(y_{i}\right)_{i<n}$ in $\prod_{i<n} F_{x_{i} \varepsilon}$, we have, using lemma 3.3,

$$
\left[s:\left(x_{i}(s)\right)_{i<n} \in R\right] \leq\left\|\left(\dot{y}_{i}\right)_{i<\check{n}} \in \check{R}^{\bullet}\right\| \leq\left[s:\left(x_{i}(s)\right)_{i<n} \in R \circ \varepsilon\right] .
$$

Since $R$ is closed, the conclusion follows. $3.9)$ :

Now we can prove the main result of this chapter (to be put together with lemma

3.10. Theorem. The map $x \mapsto \dot{x}$ is an essentially bijective map from $\mathcal{C}(S, K)$ to the class of all $B$-valued names of elements of $\bar{K}$. More precisely, this means that the following holds:

(i) $(\forall x \in \mathcal{C}(S, K))(\|\dot{x} \in \bar{K}\|=1)$;

(ii) $(\forall x, y \in \mathcal{C}(S, K))(\|\dot{x}=\dot{y}\|=1 \Rightarrow x=y)$;

(iii) $\left(\forall \boldsymbol{x} \in \mathbf{W}^{B}\right)(\|\boldsymbol{x} \in \bar{K}\|=1 \Rightarrow(\exists x \in \mathcal{C}(S, K))(\|\boldsymbol{x}=\dot{x}\|=1))$.

Proof. (i) comes from the construction of the map $x \mapsto \dot{x}$; (ii) is an immediate consequence of lemma 3.9, with $R$ being the diagonal of $K$ (we use again the fact that $K$ is Hausdorff). Finally, let $\boldsymbol{x}$ in $\mathbf{W}^{B}$ such that $\|\boldsymbol{x} \in \bar{K}\|=1$. Let $\varepsilon$ in E. By precompactness of $K$, there are $n$ in $\omega \backslash\{0\}$ and $x_{i}(i<n)$ in $K$ such that

$$
\left\|(\exists i<\check{n})\left(\left(\check{x}_{i}^{\bullet}, \boldsymbol{x}\right) \in \bar{\varepsilon}\right)\right\|=1 .
$$

For all $i<n$, let $u_{i}=\left\|\left(\check{x}_{i}^{\bullet}, \boldsymbol{x}\right) \in \bar{\varepsilon}\right\|$; then, put $v_{i}=u_{i} \wedge \neg\left(\bigvee_{j<i} u_{j}\right)$. Then $\left(v_{i}\right)_{i<n}$ is a disjoint family of elements of $B$ of join 1 , thus there exists a unique $y$ in $\mathcal{S}(S, K)$ such that

$$
(\forall i<n)\left(\forall s \in v_{i}\right)\left(y(s)=x_{i}\right)
$$

By construction, $\|(\dot{y}, \boldsymbol{x}) \in \bar{\varepsilon}\|=1$. Thus, for all $\varepsilon$ in E, the set $F_{\varepsilon}$ of all $y$ in $\mathcal{C}(S, K)$ such that $\|(\dot{y}, \boldsymbol{x}) \in \bar{\varepsilon}\|=1$ is nonempty.

Claim. The set $F_{\varepsilon}$ is closed in $\mathcal{C}(S, K)$.

Proof of claim. Let $y$ in $\mathrm{Cl}\left(F_{\varepsilon}\right)$. For every $\eta$ in E, there exists $z$ in $F_{\varepsilon}$ such that $(\forall s \in S)((y(s), z(s)) \in \eta)$, whence $\|(\dot{y}, \boldsymbol{x}) \in \mathrm{Cl}(\bar{\varepsilon})\|=1$. Since $\| \bar{\varepsilon}$ is closed $\|=1$, the conclusion follows.

- Claim . 
It follows from the claim that the intersection of all the $F_{\varepsilon}$ 's is nonempty. Pick $x$ in $\bigcap_{\varepsilon \in \mathcal{E}} F_{\varepsilon}$. Then $\|\boldsymbol{x}=\dot{x}\|=1$. This completes the proof of (iii).

Thus, we may as well replace the (proper) class of $B$-valued names of elements of $\bar{K}$ by the set of all $\dot{x}$ for $x \in \mathcal{C}(S, K)$. This reveals also the Boolean-valued interpretation of $\mathcal{C}(S, K)$ :

$\mathcal{C}(S, K)$ may be interpreted as the set of Boolean-valued names of elements of the completion of $\check{K}$ in $\mathbf{W}^{B}$.

Until now, we have worked in a universe of $Z F A$ with every element of $K$ being an atom. Now, let us work in the general context of a universe $\mathfrak{U}$ of set theory plus choice (for example $Z F C$ ), and let $K$ be a compact Hausdorff space of $\mathfrak{U}$, let $B$ be a complete Boolean algebra of $\mathfrak{U}$. Put $S=\operatorname{Ult}(B)$. Consider the first-order language $\mathcal{L}_{K}$ obtained the following way:

(i) The constant symbols of $\mathcal{L}_{K}$ are [identified with] the elements of $K$.

(ii) The relation symbols of $\mathcal{L}_{K}$ are [identified with] the closed subsets of finite powers of $K$.

(iii) The function symbols of $\mathcal{L}_{K}$ are [identified with] the continuous functions from finite powers of $K$ to itself.

Of course, $K$ and $\mathcal{C}(S, K)$ are models for $\mathcal{L}_{K}$, the interpretations of the symbols of $\mathcal{L}_{K}$ being obvious. We refer the reader to [5] for reference about first-order logic. Then, 3.9 and 3.10 allow us to derive easily the following

3.11. Corollary. $\mathcal{C}(S, K)$ is a "uniformly algebraically compact" model for $\mathcal{L}_{K}$, i.e. it is a uniformly continuous retract of each of its reduced powers.

Proof. First of all, we have to prove the

Claim. $K$ is a uniformly algebraically compact model for $\mathcal{L}_{K}$.

Proof of claim. Let ${ }^{*} K={ }^{I} K / \mathcal{F}$ be a reduced power of $K$; for all $a$ in $K$, identify $a$ with its image in ${ }^{*} K$. Consider the following system, with family of unknowns $\left(t_{\xi}\right)_{\xi \in{ }^{*} K}$ :

$$
\begin{cases}t_{a}=a & (\text { all } a \in K) \\ \left(t_{\xi_{i}}\right)_{i<n} \in A & \left(\text { all } n \text { in } \omega \backslash\{0\}, A \subseteq{ }^{n} K \text { closed, }\left(\xi_{i}\right)_{i<n} \in{ }^{*} A\right)\end{cases}
$$

Then every finite subsystem (S) of (3.1) admits a solution: in fact, there is an element $J$ of $\mathrm{F}$ such that for all $i$ in $J$, the $i^{\text {th }}$ projections of [representatives of] the elements of ${ }^{*} K$ appearing in $(\mathrm{S})$ satisfy $(\mathrm{S})$.

But the set of all solutions of any finite subsystem of (3.1) is a [topologically] closed subset of the compact ${ }^{*} K K$; thus the intersection of all these sets is nonempty. Let $\rho=\left(t_{\xi}\right)_{\xi \in{ }^{*} K}$ be an element of this set. Then $\rho$ is a retract of ${ }^{*} K$ onto $K$. It is uniformly continuous, as for every closed subset $\varepsilon$ of $K,(x, y) \in{ }^{*} \varepsilon$ (where ${ }^{*} \varepsilon$ is the natural interpretation of $\varepsilon$ in $\left.{ }^{*} K \times^{*} K\right)$ implies $(\rho(x), \rho(y)) \in \varepsilon$, in particular when $\varepsilon$ is an entourage in $K$.

- Claim . 
Now we prove our theorem. Let $\mathrm{F}$ be a filter over a nonempty set $I$; put $M=\mathcal{C}(S, K)$, let ${ }^{*} M$ be the reduced power of $M$ under F. By $\S 1$, we can assume without loss of generality that we are working in a universe $\mathbf{W}$ of $Z F A+A C$ where the set $\mathbf{A}$ of atoms contains $K$, $I$ and $S$. Equip $\mathbf{A}$ with the unique structure of $B$-scattered $B$-valued model of equality. Since $\mathbf{W}^{B}$ satisfies $Z F A+A C$, it follows from the claim that

$\| \bar{K}$ is a uniformly algebraically compact model for $\mathcal{L}_{\bar{K}} \|=1$.

Let $\mathcal{F}$ be the canonical name in $\mathbf{W}^{B}$ for the filter on $\check{I}$ generated by $\check{\mathcal{F}}$. By fullness of $\mathbf{W}^{B} \backslash \mathbf{A}$, there exists a $B$-valued name $\boldsymbol{\rho}$ such that

$$
\begin{aligned}
& \| \boldsymbol{\rho} \text { is a } \mathcal{F} \text {-invariant } \mathcal{L}_{\bar{K}} \text {-homomorphism from }{ }^{\check{I}} \bar{K} \text { to } \bar{K} \\
& \text { sending for all } \boldsymbol{x} \text { in } \bar{K} \text { the constant sequence with value } \boldsymbol{x} \text { on } \boldsymbol{x} \|=1
\end{aligned}
$$

Now, we define a map $\rho$ from ${ }^{I} M$ to $M$ the following way: given $x=\left(x_{i}\right)_{i \in I}$ in ${ }^{I} M$, let $\left(\dot{x}_{i}\right)_{i \in \check{I}}$ denote as usual the canonical $B$-valued name for the corresponding family. Then the canonical name for $\rho\left(\left(\dot{x}_{i}\right)_{i \in \check{I}}\right)$ is a $B$-valued element of $\bar{K}$, thus, by theorem 3.10, there exists a unique $x$ in $M$ such that $\left\|\dot{x}=\rho\left(\left(\dot{x}_{i}\right)_{i \in \check{I}}\right)\right\|=1$. Let this $x$ be the value of $\rho\left(\left(x_{i}\right)_{i \in I}\right)$. Then it is immediate by definition that $\rho$ is F-invariant, and that for all $x$ in $M, \rho$ sends the constant sequence with value $x$ on $x$. It remains to show that $\rho$ is a $\mathcal{L}_{K}$-homomorphism. We prove this for relation symbols, the proof for function symbols being similar. So let $n$ in $\omega \backslash\{0\}$, let $A$ be a closed subset of ${ }^{n} K$. Let $x_{k}(k<n)$ be in ${ }^{I} M$ such that

$$
(\forall i \in I)\left(\left(x_{k i}\right)_{k<n} \in \mathcal{C}(S, A)\right)
$$

where $\mathcal{C}(S, A)$ is naturally identified with the set of all $\left(x_{i}\right)_{i<n}$ in ${ }^{n} \mathcal{C}(S, K)$ such that $(\forall s \in S)\left(\left(x_{i}(s)\right)_{i<n} \in A\right)$.

For all $i$ in $I$, let $\dot{y}_{i}=\left(\dot{x}_{k i}\right)_{k<n}$. By lemma 3.9, $\left\|\dot{y}_{i} \in \bar{A}\right\|=1$, thus $\|\left(\dot{y}_{i}\right)_{i \in \check{I}} \in$ $\check{I} \bar{A} \|=1$. But $\| \bar{A}$ is a closed subset of ${ }^{\check{n}} \bar{K} \|=1$ by definition, thus, by (3.2), if we put $z_{k}=\rho\left(\left(x_{k i}\right)_{i \in I}\right)$, we have $\left\|\left(\dot{z}_{k}\right)_{k<\check{n}} \in \bar{A}\right\|=1$, whence $\left(z_{k}\right)_{k<n} \in \mathcal{C}(S, A)$ by lemma 3.9.

3.12. REMARK. It is easy to prove that in fact, uniform algebraic compactness of $K$ with respect to $\mathcal{L}_{K}$ characterizes topological compactness of $K$ (see the claim above); thus, there is no hope to prove the uniform algebraic compactness of $M=\mathcal{C}(S, K)$ with respect to the language $\mathcal{L}_{M}$ (and not just $\mathcal{L}_{K}$ ), namely because the example considered in 3.1 is not topologically compact.

3.13. EXAMPLES. Corollary 3.11 implies immediately that for every topologically compact model for a first-order theory such that relation symbols interpret closed sets and function symbols interpret continuous functions and for every extremally disconnected compact Hausdorff space $S$, the model $\mathcal{C}(S, K)$ is algebraically compact, i.e. every set of atomic formulas every finite subset of which admits a solution admits a solution. In particular, 
if $G$ is a compact Hausdorff topological group and $S$ is an extremally disconnected compact Hausdorff space, then $\mathcal{C}(S, G)$ is an algebraically compact group. Or: $\mathcal{C}(S, \overline{\mathbb{N}})$ is an algebraically compact commutative monoid. We shall prove other results of algebraic compactness in chapter 5 .

\section{$\S 4$. Case of $f$-rings.}

The previous chapter provided us with an application to the study of a structure (namely $\mathcal{C}(S, K)$ ) of a universe of $Z F A$ with a scattered set of atoms. Here and in the next chapter, the set of atoms that we will use will no longer be scattered.

Recall that a f-ring $[3$, chapitre 9$]$ is a structure $(A,+, \cdot, \wedge, \vee, 0, \leq)$ such that $(A,+, \wedge, \vee, 0, \leq)$ is a commutative $\ell$-group, $(A,+, \cdot, \leq, 0)$ is an ordered ring (not necessarily with an unit for $\cdot)$ and the following is satisfied:

$$
(\forall x, y, z \geq 0)(x \wedge y=0 \Rightarrow(x \wedge y z=x \wedge z y=0))
$$

Thus f-rings are exactly those lattice-ordered rings that are subdirect products of linearly ordered rings ( see [3, chapitre 9]). However, this representation as a subdirect product fails to carry over elementary properties of the initial structure, as e.g. the Archimedean property $(\forall x, y \geq 0)((\forall n \in \mathbb{N})(n x \leq y) \Rightarrow x=0)$.

4.1. ExAmple. The ordered ring of all [classes modulo null sets of] $\mathbb{Z}$-valued Lebesgue-measurable functions on the unit interval is an Archimedean $f$-ring. However, it is easy to prove that there is no non-trivial $\ell$-group homomorphism from this structure to the reals. Thus, its non-trivial linearly ordered components in any subdirect decomposition are not Archimedean.

We shall now see that any $f$-ring carries a canonical structure of Boolean model, similar to the subdirect decomposition mentioned above, but which, in contrast with the latter, will preserve many properties of the initial $f$-ring, as e.g. the Archimedean property (lemma 4.13).

In any $f$-ring, write as usual $x_{+}=x \vee 0, x_{-}=(-x) \vee 0,|x|=x \vee(-x)$. For all $p, q$ in $A_{+}$, let $p \mid q$ stand for $\left(\exists^{\neq 0} r\right)(r \leq p, q), p \perp q$ for $\neg(p \mid q)$, and $p \Vdash q$ for $\left(\forall^{\neq 0} r \leq p\right)(r \mid q)$. For every subset $X$ of $A$, denote by $\perp X=\{y \in A:(\forall x \in X)(|x| \perp|y|)\}$ the polar of $X$; for $p>0$, call the polar $[p]=\perp \perp p=\perp \perp\{p\}$ the carrier of $p$. If $\varphi(x)$ is any formula (with parameters), let $\left(\forall^{*} p\right) \varphi(p)$ (resp. $\left.\left(\forall^{*} q \leq p\right) \varphi(q)\right)$ stand for $\left(\forall^{\neq 0} p\right)\left(\exists^{\neq 0} q \leq p\right) \varphi(q)$ (resp. $\left.(\forall \neq 0 q \leq p)\left(\exists^{\neq 0} r \leq q\right) \varphi(r)\right)$. Define the forcing relation as follows: for all $p$ in $A_{+}$and all $a, b$ in $A$, define

$$
\left\{\begin{array}{l}
p \Vdash a \leq b \Longleftrightarrow p \perp(a-b)_{+}, \\
p \Vdash a=b \Longleftrightarrow p \perp|a-b| .
\end{array}\right.
$$

In the case where $A$ is Archimedean - more generally, in the case where all carriers are unbounded, it is shown in the proof of [13, theorem 6.6] that this definition of forcing is, for positive $a$ and $b$, equivalent to the one defined in [13, chapter 6] (and which, in 
addition, depends only on the ordering of $A$ ). However, if there exists a bounded carrier, then both definitions of forcing are never equivalent.

Now, let $B=\mathcal{B}(A)$ be the complete Boolean algebra of polar subsets of $A_{+}$; thus $u \in B$ if and only if $u \backslash\{0\}$ is a regular-open subset of $P=A_{+} \backslash\{0\}$ equipped with its "left-topology" (for which a basis of open sets is the set of all $P_{p}=\{q \in P: q \leq p\}$, $p \in P$ ). Thus $p \mapsto[p]$ is the natural map from $P$ to $B$. For all $a, b$ in $A$, put

$$
\left\{\begin{array}{l}
\llbracket a \leq b \rrbracket=\bigvee\{[p]: p \Vdash a \leq b\}, \\
\llbracket a=b \rrbracket=\bigvee\{[p]: p \Vdash a=b\} .
\end{array}\right.
$$

4.2. Lemma. Let $p$ in $A_{+}$, let $a, b$ in $A$. Then the following are equivalent:

(i) $p \Vdash a \leq b$;

(ii) $\left(\forall^{*} q \leq p\right)(q \Vdash a \leq b)$;

(iii) $[p] \leq \llbracket a \leq b \rrbracket$,

and the following are equivalent:

(i') $p \Vdash a=b$;

(ii') $\left(\forall^{*} q \leq p\right)(q \Vdash a=b)$;

(iii') $[p] \leq \llbracket a=b \rrbracket$.

Proof. Easy.

Our basic tool will be the following

4.3. Proposition. A, equipped with the Boolean-value functions defined in (4.1) and (4.2), is a $B$-valued model for the language $(=,+, \cdot \wedge, \vee, 0, \leq)$ of the theory of linearly ordered rings. Furthermore, for all $a, b$ in $A, a \leq b$ (resp. $a=b$ ) if and only if $\llbracket a \leq b \rrbracket=1$ (resp. $\llbracket a=b \rrbracket=1)$.

Proof. First of all, it is easy to check that for all $p$ in $A_{+}$and all $a, a^{\prime}, b, b^{\prime}, c$ in $A$, the following holds:

$$
\left\{\begin{array}{l}
p \Vdash a=a ; \quad p \Vdash a=b \Leftrightarrow p \Vdash b=a ; \\
(p \Vdash a=b \text { and } p \Vdash b=c) \Rightarrow p \Vdash a=c ; \\
\left(p \Vdash a \leq b \text { and } p \Vdash a=a^{\prime} \text { and } p \Vdash b=b^{\prime}\right) \Rightarrow p \Vdash a^{\prime} \leq b^{\prime} ; \\
\left(p \Vdash a=a^{\prime} \text { and } p \Vdash b=b^{\prime}\right) \Rightarrow p \Vdash a+b=a^{\prime}+b^{\prime} ; \\
\left(p \Vdash a=a^{\prime} \text { and } p \Vdash b=b^{\prime}\right) \Rightarrow p \Vdash a \cdot b=a^{\prime} \cdot b^{\prime}
\end{array}\right.
$$

Note that the last property listed in (4.3) uses the fact that $A$ is a $f$-ring.

Thus, similar relations hold for the Boolean values defined in (4.2) $(\llbracket a=b \rrbracket=\llbracket b=a \rrbracket$, etc.), and $A$ is a $B$-valued model of the language $(=, \leq,+, \cdot)$; thus, we can define the Boolean value of any formula of this language (with parameters in $A$ ) in a way that preserves truth (see e.g. [11], [12]). For $\wedge$ and $\vee$, we could prove similar properties as the last two in (4.3), but they result from the following 
Claim. Let $a, b$ in $A$, let $c=a \wedge b$ and $d=a \vee b$. Then

$$
\llbracket c=\inf (a, b) \text { for } \leq \rrbracket=\llbracket d=\sup (a, b) \text { for } \leq \rrbracket=1 \text {. }
$$

Proof of claim. Since $c \leq a, b$, we have $p \Vdash c \leq a$ and $p \Vdash c \leq b$ for all $p$ in $A_{+}$. Furthermore, let $x$ in $A$ such that $p \Vdash x \leq a$ and $p \Vdash x \leq b$. This means that $p \perp(x-a)_{+}$ and $p \perp(x-b)_{+}$, whence $p \perp(x-a)_{+} \vee(x-b)_{+}=(x-c)_{+}$, i.e. $p \Vdash x \leq c$. Thus the first assertion follows. The proof for $d$ is similar.

- Claim .

Furthermore, it is easy to verify that the Boolean value of the statement saying that the model is a partial ordering is equal to 1. Then, the claim implies that the Boolean value of the statement saying that the model is a lattice is equal to 1 . Moreover, it is easy to check the following facts:

$$
\left\{\begin{array}{l}
\left(p \Vdash a \leq a^{\prime} \text { and } p \Vdash b \leq b^{\prime}\right) \Rightarrow p \Vdash a+b \leq a^{\prime}+b^{\prime} \\
(p \Vdash a \leq b \text { and } p \Vdash c \geq 0) \Rightarrow(p \Vdash a \cdot c \leq b \cdot c \text { and } p \Vdash c \cdot a \leq c \cdot b) \\
p \Vdash a \leq b \Leftrightarrow p \Vdash(\exists x \geq 0)(a+x=b) \Leftrightarrow p \Vdash b-a \geq 0
\end{array}\right.
$$

Thus the Boolean value of the statement saying that the model is a lattice-ordered ring is equal to 1 .

Let $a, b$ in $A$, let $p$ in $A_{+} \backslash\{0\}$. If $p \| \forall a \not \leq b$, then there exists $q \leq p$ non zero such that $q \Vdash a \leq b$. If on the contrary $p \Vdash a \not \leq b$, then $p \Vdash(a-b)_{+}$, thus $p \perp(b-a)_{+}$, whence $p \Vdash b \leq a$ : thus, $\llbracket a \leq b$ or $b \leq a \rrbracket=1$.

It remains to check that $\llbracket a \leq b \rrbracket=1$ implies $a \leq b$ (the conclusion for $=$ follows). If $\llbracket a \leq b \rrbracket=1$ but $a \not \leq b$, then there exists $p \leq(a-b)_{+}$non zero such that $p \Vdash a \leq b$, i.e. $p \perp(a-b)_{+}$, whence $p=0$ since $p \leq(a-b)_{+}$, a contradiction. Thus $a \leq b$.

Note in particular that for all $p$ in $A_{+}$and $a, b$ in $A$ such that $p \Vdash a \leq b$, we have $p \Vdash a+c=b$ where $c=(b-a)_{+}$: this is because $\llbracket c=(b-a) \vee 0 \rrbracket=1$, but $[p] \leq \llbracket a \leq b \rrbracket$ whence $[p] \leq \llbracket a+c=b \rrbracket$.

The notion of convergence that we shall present now is similar to the one in $[13$, chapter 7]. Recall that a net is a family indexed by some directed set.

4.4. Definition. Let $\left(x_{\nu}\right)_{\nu \in \mathcal{D}}$ be a net with values in $A$, let $x$ in $A$. Then we will write that $x=\lim _{\nu \in \mathcal{D}} x_{\nu}$ when for all $p$ in $A_{+} \backslash\{0\}$ and all $a, b$ in $A$, the following holds:

$$
\begin{aligned}
& \text { If } p \Vdash a<x \text { then }\left(\exists^{\neq 0} q \leq p\right)\left(\forall^{\infty} \nu\right)\left(q \Vdash a<x_{\nu}\right) \text {; } \\
& \text { If } p \Vdash x<b \text { then }\left(\exists^{\neq 0} q \leq p\right)\left(\forall^{\infty} \nu\right)\left(q \Vdash x_{\nu}<b\right) \text {. }
\end{aligned}
$$

It is important to note that if we work in a universe of $Z F A$ with elements of $A$ being atoms and Boolean valued defined as in (4.1) and (4.2) such that D is B-scattered (see 2.5, (7)), then $x=\lim _{\nu \in \mathcal{D}} x_{\nu}$ is equivalent to the statement

$$
\| x=\lim _{\nu \in \mathcal{D}} x_{\nu} \text { for the interval topology on } \check{A} \|=1 \text {. }
$$


But in linearly ordered spaces, topological limit (for the interval topology) is a generalization of [left-] directed infimum and [right-] directed supremum. The following proposition, stated in a universe of $Z F A$ where elements of $A$ are atoms, carries over this fact to our general context:

4.5. Proposition. Let $a \in A$ and $X \subseteq A$ nonempty. Then we have

(i) $a=\bigwedge X$ if and only if $\|a=\bigwedge \check{X}\|=1$;

(ii) $a=\bigvee X$ if and only if $\|a=\bigvee \check{X}\|=1$.

Proof. (i) If $a=\bigwedge X$, then $\|a \leq \check{X}\|=1$. Let $b$ in $A$, we have to show that $\|b \leq \check{X}\| \leq\|b \leq a\|$. So let $p$ in $A_{+} \backslash\{0\}$ such that $[p] \leq\|b \leq X\|$, i.e., by lemma 4.2, $p \Vdash b \leq x$ for all $x$ in $X$. Then $\bigvee_{x \in X}(b-x)_{+}$is defined and equal to $(b-a)_{+}$, thus $p \perp(b-a)_{+}$, i.e. $p \Vdash b \leq a$. So we have proved that $\|a=\bigwedge \check{X}\|=1$. Conversely, suppose that $\|a=\bigwedge \check{X}\|=1$. Then for all $x$ in $X,\|a \leq x\|=1$, thus $a \leq x$ by proposition 4.2. Let $b$ in $A$ such that $b \leq X$; then $\|b \leq \check{X}\|=1$, whence $\|b \leq a\|=1$ by assumption, whence $b \leq a$. Thus $a=\bigwedge X$.

The proof for (ii) is similar.

4.6. Corollary. Let $\left(x_{\nu}\right)_{\nu \in \mathcal{D}}$ be a $A$-valued net, let $x$ in $A$. If $\left(x_{\nu}\right)_{\nu \in \mathcal{D}}$ is increasing (resp. decreasing), then $x=\bigvee_{\nu \in \mathcal{D}} x_{\nu}$ (resp. $x=\bigwedge_{\nu \in \mathcal{D}} x_{\nu}$ ) if and only if $x=\lim _{\nu \in \mathcal{D}} x_{\nu}$.

Our first application of the forcing (4.1) will be the following

4.7. Theorem. Let $P$ be a polynomial with one indeterminate $t$ with coefficients in a commutative $f$-ring $A$, that is, $P \in A[t]$. Then the graph of $P$ is closed with respect to the previously defined notion of convergence, i.e. if $\left(x_{\nu}\right)_{\nu \in \mathcal{D}}$ is a $A$-valued net, $a$ and $b$ are in $A$ and $\lim _{\nu \in \mathcal{D}} x_{\nu}=a$ and $\lim _{\nu \in \mathcal{D}} P\left(x_{\nu}\right)=b$, then $b=P(a)$.

Proof. We start by reducing the proof to the linearly ordered case; so suppose that we are done in the linearly ordered case. In the general case, we can by $\S 1$ without loss of generality assume that we work in a universe of $Z F A$ with set of atoms containing $A$ and $\mathrm{D}$ (with $A$ and $\mathrm{D}$ made disjoint), $\mathrm{D}$ being $B$-scattered and $A$ being equipped with its natural structure of $B$-valued model defined in (4.1) and (4.2). Then, using proposition 4.3, $\left\|a=\lim _{\nu \in \check{\mathcal{D}}} x_{\nu}\right\|=\left\|b=\lim _{\nu \in \check{\mathcal{D}}} P\left(x_{\nu}\right)\right\|=1$; but $\| \check{A}$ is linearly ordered $\|=1$, whence $\|b=P(a)\|=1$ by assumption, whence $b=P(a)$ again by proposition 4.3.

Now, suppose that $A$ is linearly ordered, and let us prove directly the conclusion of the theorem. Since $A$ is linearly ordered, we may assume without loss of generality that $\lim _{\nu \in \mathcal{D}} x_{\nu}=0$, that $x_{\nu}>0$ for all $\nu$ (thus 0 is not an isolated point in $A$ ), that $P(0)=0$ and that $P \neq 0$. Thus there are $n$ in $\omega \backslash\{0\}$ and $a_{1}, \ldots, a_{n}$ in $A$, with $a_{n} \neq 0$, such that $P(t)=a_{1} t+\cdots+a_{n} t^{n}$.

If $A$ is not a domain, then there are strictly positive $u, v$ such that $u v=0$; taking $c=u \wedge v$, we have $c>0$ and $c^{2}=0$, thus, for all $x$ in $[0, c]$, we have $P(x)=a_{1} x$. Without loss of generality, $a_{1} \geq 0$. Since $A$ is linearly ordered, for all $\nu$, there exists $\nu^{\prime} \geq \nu$ such that $2 x_{\nu^{\prime}} \leq x_{\nu}$, whence $2 b \leq 2 P\left(x_{\nu^{\prime}}\right)=P\left(2 x_{\nu^{\prime}}\right) \leq P\left(x_{\nu}\right)$; thus $2 b \leq b$, but $b \geq 0$, whence $b=0$. So it remains to prove this conclusion in the case where $A$ is a domain. 
For all $x$ and $y$ in $A$, say that $x$ has magnitude less than $y$ when $(\exists n \in \mathbb{N})(|x| \leq n|y|)$; let $\bar{x}$ denote the magnitude of $x$. Put $I=\left\{i \in[1, n]: a_{i} \neq 0\right\}$. For each $x \neq 0$, let $h(x)$ be the unique element $k$ of $I$ such that

$$
\begin{aligned}
& (\forall i \in I)\left(i<k \Rightarrow \overline{a_{i} x^{i}}<\overline{a_{k} x^{k}}\right) \\
& (\forall i \in I)\left(i \geq k \Rightarrow \overline{a_{i} x^{i}} \leq \overline{a_{k} x^{k}}\right)
\end{aligned}
$$

Claim 1. $0<\bar{y} \leq \bar{x}$ implies $h(y) \leq h(x)$.

Proof of claim. Put $k=h(x)$ and $l=h(y)$, and suppose that $k<l$. Since $A$ is a domain, we have $\overline{a_{k}}<\overline{a_{l} y^{l-k}}$ and $\overline{a_{l} x^{l-k}} \leq \overline{a_{k}}$, whence $\overline{a_{l} x^{l-k}}<\overline{a_{l} y^{l-k}}$, contradicts $\bar{y} \leq \bar{x}$.

- Claim 1.

Now, let $k$ the least element of $\{h(x): x>0\}$, and let $b>0$ such that $h(b)=k$. By claim $1, h(x)=k$ for all $x$ in $(0, b]$.

Claim 2. Let $N$ in $\mathbb{N} \backslash\{0\}$. Then there exists $c$ in $(0, b]$ such that

$$
(\forall x \in(0, c])\left(N \cdot\left|P(x)-a_{k} x^{k}\right| \leq\left|a_{k} x^{k}\right|\right) .
$$

Proof of claim. Put $Q(t)=P(t)-a_{k} t^{k}$. Since $h(b)=k$, there exists $m$ in $\mathbb{N} \backslash\{0\}$ such that

$$
(\forall i>k)\left(\left|a_{i} b^{i}\right| \leq m \cdot\left|a_{k} b^{k}\right|\right) .
$$

Since 0 is not isolated, there exists $c>0$ such that $0<m n N c \leq b$. Thus for all $i>k$ and all $x$ in $(0, c]$, we have

$$
(m n N) \cdot\left|a_{i} x^{i-k}\right| \leq(m n N)^{i-k}\left|a_{i} x^{i-k}\right| \leq\left|a_{i}((m n N) c)^{i-k}\right| \leq\left|a_{i} b^{i-k}\right| \leq m \cdot\left|a_{k}\right|,
$$

whence $n N \cdot\left|a_{i} x^{i}\right| \leq\left|a_{k} x^{k}\right|$. But this also holds for $i<k$ by definition of $k$. Thus, it follows that

$$
n N \cdot|Q(x)| \leq(n-1) \cdot\left|a_{k} x^{k}\right| \leq n \cdot\left|a_{k} x^{k}\right|,
$$

whence the conclusion of the claim follows.

- Claim 2.

Now, we can assume without loss of generality that $a_{k}>0$. By claim 2, there exists $c>0$ such that

$$
(\forall x \in(0, c])\left(3^{k+1}|Q(x)| \leq a_{k} x^{k}\right) .
$$

Claim 3. Let $x, y$ such that $0<3 y \leq x \leq c$. Then $P(2 y) \leq P(x)$.

Proof of claim. Let $z=x-2 y$. Thus $3 y \leq x=2 y+z$ whence $y \leq z$, whence $x \leq 3 z$. But by definition of $c$, we have

$$
2 \cdot 3^{k} \cdot|Q(x)-Q(2 y)| \leq a_{k} x^{k}+a_{k}(2 y)^{k} \leq 2 a_{k} x^{k} \leq 2 \cdot 3^{k} \cdot a_{k} z^{k},
$$

whence

$$
|Q(x)-Q(2 y)| \leq a_{k} z^{k} \leq a_{k} x^{k}-a_{k}(2 y)^{k} .
$$

The conclusion follows.

- Claim 3. 
Claim 4. Let $x$ such that $0<2 x \leq c$. Then $2 P(2 x) \geq 3 P(x)$.

Proof of claim. We have

$$
\begin{aligned}
P(2 x) & =2^{k} a_{k} x^{k}+Q(2 x) \\
& =2^{k} P(x)+\left(Q(2 x)-2^{k} Q(x)\right),
\end{aligned}
$$

thus, taking $N=3^{k+1}$ and by definition of $c$,

$$
N \cdot P(2 x) \geq 2^{k} N \cdot P(x)-2^{k+1} a_{k} x^{k} .
$$

But $(N-1) a_{k} x^{k} \leq N \cdot P(x)$, whence

$$
\begin{aligned}
(N-1) N \cdot P(2 x) & \geq 2^{k}(N-1) N \cdot P(x)-2^{k+1} N \cdot P(x) \\
& =2^{k} N(N-3) \cdot P(x)
\end{aligned}
$$

thus $(N-1) \cdot P(2 x) \geq 2^{k}(N-3) \cdot P(x) \geq(2 N-6) \cdot P(x)$, whence it follows easily that $2 P(2 x) \geq 3 P(x)$.

- Claim 4.

Now we can conclude. Without loss of generality, we have $(\forall \nu \in \mathcal{D})\left(0<x_{\nu} \leq c\right)$. For all $\nu$ in $\mathrm{D}$, there exists $\nu^{\prime} \geq \nu$ in $\mathrm{D}$ such that $3 x_{\nu^{\prime}} \leq x_{\nu}$. Therefore, by claim $3, P\left(2 x_{\nu^{\prime}}\right) \leq$ $P\left(x_{\nu}\right)$; furthermore, by claim $4,3 P\left(x_{\nu^{\prime}}\right) \leq 2 P\left(2 x_{\nu^{\prime}}\right)$, whence $3 b \leq 3 P\left(x_{\nu^{\prime}}\right) \leq 2 P\left(x_{\nu}\right)$. Thus $3 b \leq 2 b$, but $b \geq 0$ thus $b=0$, which completes the proof.

4.8. EXAmPle. Let $A=\mathbb{R}[t]$ be the polynomial ring with one indeterminate over $\mathbb{R}$; order $A$ by keeping the natural order over $\mathbb{R}$ and by making $t$ infinitely large (with respect to $\mathbb{R}$ ). Put $x_{n}=1 / n$ for all $n$ in $\mathbb{N} \backslash\{0\}$. Then $\lim _{n \in \mathbb{N} \backslash\{0\}} x_{n}=0$, while the sequence $\left(x_{n} t\right)_{n \in \mathbb{N} \backslash\{0\}}$ is not convergent.

4.9. EXAMPLE. Theorem 4.7 has been stated for polynomials in one indeterminate; it is no longer valid for general $\ell$-polynomials, that is the terms of the language $(+, \cdot, \wedge, \vee)$. For example, let us consider the linearly ordered ring $\mathbb{R}[t]$ of previous example, and the same sequence $\left(x_{n}\right)_{n \in \mathbb{N} \backslash\{0\}}$. Put $P(x)=(1-t x) \vee 0$. Then $\lim _{n \in \mathbb{N} \backslash\{0\}} x_{n}=0$ and $\lim _{n \in \mathbb{N} \backslash\{0\}} P\left(x_{n}\right)=0$, while $P(0)=1$.

4.10. EXAmPLE. Still for commutative linearly ordered rings, another generalization of theorem 4.7 that does not hold is by considering polynomials with 2 variables. An example of such a situation is the following. Let $\lambda$ be a positive real number which is transcendant over $\mathbb{Q}$, let $K=\mathbb{Q}[\lambda]$. Let $\left(t_{n}\right)_{n \in \omega}$ be an algebraically independent over $K$ sequence of real numbers which decreases to 0 , and put finally $A=K\left[t, \frac{1}{t}+\lambda t_{n}\right]_{n \in \omega}$ where $t$ is an indeterminate. Extend the usual linear ordering of $K$ by saying that $t$ is infinitely large (and ordering lexicographically with respect to the increasing powers of $t$ ). Thus $A$ is a linearly ordered commutative ring. Say that an element $\alpha$ of $A$ is infinitesimal when $(\forall n \in \mathbb{N} \backslash\{0\})(|\alpha| \leq 1 / n)$. Then it is not difficult to check that 0 is the only infinitesimal 
element of $A$. Now, for all $n$ in $\omega$, put $x_{n}=\frac{1}{t}+\lambda t_{n}$. Since 0 is the only infinitesimal element of $A,\left(x_{n}\right)_{n}$ and $\left(t_{n}\right)_{n}$ are two decreasing sequences of elements of $A$, with limit 0 . But for all $n$, we have $t x_{n}-(\lambda t) t_{n}=1$ (with $t, \lambda t, x_{n}, t_{n}$ in $A$ ). Thus if $P(x, y)$ is the polynomial $t x-(\lambda t) y$, then $P \in A[x, y],\left(x_{n}\right)_{n}$ and $\left(t_{n}\right)_{n}$ converge to 0 in $A, P(0,0)=0$, but $\lim _{n \rightarrow \infty} P\left(x_{n}, t_{n}\right)=1$.

4.11. ExAmple. The following example shows what happens with polynomials over a non-commutative linearly ordered ring. Let $R$ be a ring, let $\partial$ be a derivation on $R$, i.e. an additive endomorphism of $R$ satisfying the property

$$
(\forall x, y \in R)(\partial(x \cdot y)=x \cdot \partial(y)+\partial(x) \cdot y)
$$

Let $x$ denote an indeterminate over $R$, and equip $R[x]$ with its usual addition; it is not a difficult exercise to verify that there exists a unique associative multiplication on $R[x]$ which is distributive with respect to + such that for all $a$ in $R, a x-x a=\partial(a)$. Now, let $R$ be the subring of $\mathbb{R}$ generated by the rationals and some algebraically independent sequence $\left(\alpha_{n}\right)_{n \in \omega}$ of positive numbers such that $\lim _{n \rightarrow \infty} \alpha_{n}=0$. Equip $R$ with the derivation $\partial$ defined by

$$
\partial(P(\vec{\alpha}))=\sum_{n \in \omega} \frac{\partial P}{\partial x_{n}}(\vec{\alpha}) .
$$

It is easy to verify that $\partial$ is a derivation on $R$. Consider the ring $R[x]$ as before, equipped with the unique linear ring-ordering extending the natural ordering of $R$ and making $x$ infinitely large. Put $P(t)=t x-x t$. Then for all $n, P\left(\alpha_{n}\right)=\alpha_{n} x-x \alpha_{n}=\partial \alpha_{n}=1$, while $\lim _{n \rightarrow \infty} \alpha_{n}=0$ and $P(0)=0$. Thus theorem 4.7 is not valid in arbitrary non-commutative linearly ordered rings.

Still, there exists an analogue of theorem 4.7 for $\ell$-polynomials with several variables. To state it, introduce the class of positive $\ell$-polynomials to be the least class of $\ell$ polynomials containing the elementary polynomials $P\left(\left(x_{i}\right)_{i<n}\right)=x_{j}$ ar $P\left(\left(x_{i}\right)_{i<n}\right)=a x_{j}$ ( $a \in A_{+}, j<n$ fixed), and such that if $P$ and $Q$ are positive $\ell$-polynomials, then so are $P+Q, P \cdot Q, P \wedge Q$ and $P \vee Q$.

4.12. Proposition. Let $P\left(\left(x_{i}\right)_{i<n}\right)$ be a positive $\ell$-polynomial on a $f$-ring $A$, let $X$ be a left-directed subset of ${ }^{n} A_{+}$such that $\bigwedge X=0$ and $\bigwedge P[X]$ is defined. Then $\bigwedge P[X]=0$.

Proof. As in the proof of theorem 4.7, it suffices to prove the theorem in the case where $A$ is linearly ordered. Let $a=\bigwedge P[X]$. Since $P$ is positive, it is easy to verify that $P$ defines an increasing function from ${ }^{n} A_{+}$to $A_{+}$such that for all $\vec{x}$ in ${ }^{n} A_{+}$, we have $P(2 \vec{x}) \geq 2 P(\vec{x})$. Now, for all $\vec{x}$ in $X$, the hypotheses imply that there exists $\vec{y}$ in $X$ such that $2 \vec{y} \leq \vec{x}$. Thus $2 a \leq 2 P(\vec{y}) \leq P(2 \vec{y}) \leq P(\vec{x})$, whence $2 a \leq a$. Thus $a=0$.

In particular, if $n \in \mathbb{N} \backslash\{0\}$ and $a, b, c \in A_{+}$and $X$ is any subset of $A_{+}$such that $\bigwedge X=b$ and $\bigwedge a X^{n}=c$ (where $a X^{n}=\left\{a x^{n}: x \in X\right\}$ ), then $c=a b^{n}$ (and similarly for the polynomial $\left.x^{n} a\right)$ : the reason is that the polynomial $(a+y)^{n}-a^{n}$ is a positive 
polynomial, to which the previous proposition applies, and that for all $x, y$ in $A_{+}$, we have $a x^{n} \wedge a y^{n}=a(x \wedge y)^{n}($ see $[3])$.

In the case where the ring is Archimedean, all the problems of existence of limits vanish, since the following lemma reduces the problem to the real case:

4.13. Lemma. Let $A$ be an Archimedean f-ring. Then $\| \check{A}$ is Archimedean $\|=1$.

Proof. We have to prove that for all $a, b$ in $A_{+}, \bigwedge_{n \in \mathbb{N}}\|n a \leq b\| \leq\|a=0\|$. So let $p$ in $A_{+} \backslash\{0\}$ such that $[p]$ is less or equal to the left-hand side. By lemma 4.2, for all $n$ in $\mathbb{N}, p \Vdash n a \leq b$, whence $n p \perp(n a-b)_{+}$. It follows that

$$
(n p-b) \wedge(n a-b) \leq n p \wedge(n a-b)_{+} \leq 0
$$

whence $n p \wedge n a \leq b$, i.e. $n(p \wedge a) \leq b$. Thus, since $A$ is Archimedean, $p \wedge a=0$, i.e. $p \Vdash a=0$. The conclusion follows.

4.14. Corollary. Let $A$ be an Archimedean f-ring. Then every $\ell$-polynomial on $A$ is continuous with respect to the above notion of convergence, i.e. for all $n$ in $\omega \backslash\{0\}$, every $\ell$-polynomial $P$ with $n$ indeterminates, every $\vec{x}$ in ${ }^{n} A$ and every ${ }^{n} A$-valued net $\left(x_{\nu}\right)_{\nu \in \mathcal{D}}$, $\vec{x}=\lim _{\nu \in \mathcal{D}} \vec{x}_{\nu}$ implies $P(\vec{x})=\lim _{\nu \in \mathcal{D}} P\left(\vec{x}_{\nu}\right)$.

Proof. Using lemma 4.13, one sees once again that it suffices to prove the theorem when $A$ is linearly ordered. Then, $A$ is isomorphic to a linearly ordered ring consisting on an additive subgroup of $\mathbb{R}$, equipped with the multiplication defined by $(x, y) \mapsto c x y$ for some $c \geq 0$. But then $P$ defines a topologically continuous function (for the interval topology), whence the conclusion follows.

This result contains in particular the classical result that for all $a \geq 0$ and all $X \subseteq A$ such that $\bigwedge X$ is defined, then $\bigwedge(a \cdot X)$ is defined and equal to $a \cdot \bigwedge X-$ it suffices to apply corollary 4.14 to the set of finite meets of elements of $X$ ( see [3, chapitre 12]).

\section{$\S 5$. Algebraic compactness of ordered structures.}

We shall assume through this chapter that the axiom of choice is satisfied.

Let $\mathrm{L}$ be a first-order language, let $\mathfrak{M}$ be a model for $\mathrm{L}$. An atomic L-system with parameters from $\mathfrak{M}$ is by definition a set $\Gamma$ of atomic formulas of $L$ with parameters from $\mathfrak{M}$; note that we put no restriction on the set $I$ of all variables occurring in all the formulas of $\Gamma$. If $M$ is the underlying set of $\mathfrak{M}$ and $\vec{x} \in{ }^{I} M$, we will say that $\vec{x}$ is a solution of $\Gamma$ when for all $\varphi$ in $\Gamma, \mathfrak{M}$ satisfies $\varphi(\vec{x}) ; \Gamma$ is solvable in $\mathfrak{M}$ when it admits a solution in ${ }^{I} M$. We will say that $\mathfrak{M}$ is algebraically compact (relatively to the language $\mathrm{L}$ ) when for every atomic L-system $\Gamma$ with parameters from $\mathfrak{M}$, if $\Gamma$ is finitely solvable (i.e. every finite subset of $\Gamma$ is solvable), then $\Gamma$ is solvable. This definition is similar to the classical definition of equational compactness (see [16]), or algebraic compactness for abelian groups (see [6]), but instead of just considering equations, we consider arbitrary atomic formulas - a typical example is in chapter 3 , for the $\mathcal{C}(S, K)$ 's; no extra complication arises from this generalization. Actually, in all the cases considered in this chapter, where the ordering 
$\leq$ can be defined by $x \leq y \Leftrightarrow(\exists z \geq 0)(x+z=y)$, equational compactness and algebraic compactness are easily seen to be equivalent.

A large part of this chapter will be concerned with algebraic compactness properties of $f$-rings.

5.1. Definition. Let $A$ be a commutative $\ell$-group. We say that $A$ is locally full when for every antichain $W$ of $A_{+}$and every bounded family $\left(x_{p}\right)_{p \in W}$ of elements of $A$ (i.e. $\left.\left(\exists a \in A_{+}\right)(\forall p \in W)\left(\left|x_{p}\right| \leq a\right)\right)$, there exists $x$ in $A$ such that $(\forall p \in W)\left(p \Vdash x=x_{p}\right)$ see $(4.1))$.

When every element of $A$ is an atom of the universe and $A$ is equipped with its structure of Boolean model described in (4.2), local fullness of $A$ is of course equivalent to the fact that for every $B$-valued name $\boldsymbol{x}$ (where $B$ is the complete Boolean algebra of polar subsets of $A$ ) and every $a$ in $A_{+},\|\boldsymbol{x} \in \check{A}\|=1$ and $\||\boldsymbol{x}| \leq a\|=1$ implies that there exists $x$ in $A$ such that $\|\boldsymbol{x}=x\|=1$.

Say that a subset $X$ of $A$ is a direct factor in $A$ when $A=X+\perp X$; this implies that $X$ is a polar set. Then, for each $x$ in $A$, denote by $x \uparrow X$ the projection of $x$ on $X$ along $\perp X$. When $X=\perp \perp p(p \geq 0)$, then just write $x\lceil p$. Recall (see [3]) that $A$ is projectable when for all $p$ in $A_{+}, A=\perp p+\perp \perp p$ (i.e. $\perp p$ is a direct factor in $A$ ). Say that $A$ is conditionally laterally complete when for every bounded antichain $W$ of elements of $A_{+}, \bigvee W$ exists. Local fullness admits a simple equivalent in terms of these definitions:

5.2. Lemma. Let $A$ be a commutative $\ell$-group. Then the following are equivalent:

(i) $A$ is locally full;

(ii) $A$ is projectable and conditionally laterally complete;

(iii) $A$ is conditionally laterally complete and every polar of $A$ is a direct factor.

Proof. (i) $\Rightarrow$ (ii) Assume (i). Let $a, b$ in $A_{+}, b \neq 0$. Let $W$ be a maximal antichain of elements of $A_{+} \backslash\{0\}$ such that $b \in W$. Define families $\left(x_{p}\right)_{p \in W}$ and $\left(y_{p}\right)_{p \in W}$ by $x_{b}=a$, $x_{p}=0$ for $p \neq b, y_{b}=0, y_{p}=a$ for $p \neq b$. By assumption, there are $x$ and $y$ in $A$ such that $(\forall p \in W)\left(p \Vdash x=x_{p}\right.$ and $\left.p \Vdash y=y_{p}\right)$. It is easy to verify that $x \in \perp \perp b, y \in \perp b$ and $x+y=a$.

(ii) $\Rightarrow$ (iii) Assume (ii). Let $X$ be a polar subset of $A$. Let $W$ be a maximal antichain of elements of $A_{+} \backslash\{0\}$ such that $(\forall p \in W)(p \in X$ or $p \in \perp X)$. Let $x$ in $A_{+}$; then $y=\bigvee\{x\lceil p: p \in W \cap X\}$ and $z=\bigvee\{x\lceil p: p \in W \cap \perp X\}$ exist, and $y \in X$ and $z \in \perp X$ and $y+z=x$.

(iii) $\Rightarrow$ (ii) is trivial.

(ii) $\Rightarrow$ (i) Assume (ii). Let $W$ be an antichain of $A_{+}$, let $a$ in $A_{+}$, let $\left(x_{p}\right)_{p \in W}$ be a family of elements of $A$ such that $(\forall p \in W)\left(\left|x_{p}\right| \leq a\right)$. Then both $x^{\prime}=\bigvee_{p \in W}\left(\left(x_{p}\right)_{+}\lceil p)\right.$ and $x^{\prime \prime}=\bigvee_{p \in W}\left(\left(x_{p}\right)_{-}\lceil p)\right.$ exist; let $x=x^{\prime}-x^{\prime \prime}$, then we have $(\forall p \in W)\left(p \Vdash x=x_{p}\right)$.

In particular, every complete $\ell$-group is locally full. In fact, a slight modification of the proof in [2] shows that every conditionally laterally $\sigma$-complete Archimedean $\ell$-group is projectable, thus that every conditionally laterally complete Archimedean $\ell$-group is locally full. 
The following lemma gives us a characterization of projectability of the Boolean model associated with a given commutative $\ell$-group $A$ in terms of polar subsets of $A$ :

5.3. Lemma. Let $A$ be a commutative $\ell$-group, equipped with its notion of forcing defined in (4.1) and (4.2); assume that the elements of $A$ are atoms of the universe. Then the following are equivalent:

(i) $A$ is a projectable Boolean model (as in definition 2.8);

(ii) Every polar subset of $A$ is a direct factor of $A$.

Proof. (i) $\Rightarrow$ (ii) Assume (i). Let $u$ in $B$. For each $x$ in $A$, there exists, by assumption, $y$ in $A$ such that $u \leq\|y=x\|$ and $\neg u \leq\|y=0\|$, i.e. $y \in u$ and $x-y \in \perp u$, whence $A=u+\perp u$.

(ii) $\Rightarrow$ (i) Suppose (ii) satisfied, let $x, y$ in $A$ and $u$ in $B$. Let $z=x\lceil u+y \uparrow \neg u$. It is immediate that $u \leq\|z=x\|$ and $\neg u \leq\|z=y\|$.

In particular, when $A$ is a complete $\ell$-group, $A$ is a projectable $B$-valued model. Thus, we deduce the

5.4. Lemma. Let $A$ be a complete f-ring, equipped with its notion of forcing defined in (4.1) and (4.2); assume that the elements of $A$ are atoms of the universe. Then $\| \check{A}$ is a complete linearly ordered ring $\|=1$.

Proof. We have seen in proposition 4.3 that $\| \check{A}$ is a linearly ordered ring $\|=1$. It remains to prove that $\| \check{A}$ is complete $\|=1$. By lemma $5.4, A$ is a projectable $B$-valued model, thus it suffices by lemma 2.10 to prove that for all $X \subseteq A$, we have

$$
\| \check{X} \text { is bounded nonempty } \Rightarrow \bigwedge \check{X} \text { exists } \|=1 \text {. }
$$

For $X=\emptyset$ it is trivial; so suppose $X \neq \emptyset$. To prove (5.1), it suffices then to prove that for all $a$ in $A_{+}$, we have

$$
\|-a \leq \check{X} \leq a\| \leq \| \bigwedge \check{X} \text { exists } \|
$$

Let $u$ be the left-hand side of (5.2). By proposition 2.12, there exists $Y$ in $\mathcal{P}(A) \backslash\{\emptyset\}$ such that $u \leq\|\check{Y}=\check{X}\|$ and $\neg u \leq\left\|\check{Y}=\{0\}^{`}\right\|$. Thus $\|-a \leq \check{Y} \leq a\|=1$, whence $-a \leq Y \leq a$ by proposition 4.3. Hence $Y$ is bounded nonempty, whence $b=\bigwedge Y$ exists. By proposition 4.5 , we have $\|b=\bigwedge \check{Y}\|=1$, whence $u \leq\|b=\bigwedge \check{X}\|$, which completes the proof of (5.2).

Now we are in position to prove the

5.5. Theorem. Let $A$ be a complete $f$-ring. Consider an atomic system of the following form:

$$
\begin{cases}\varphi_{i}(\vec{x}) & \left(\text { all } i \in I ; \text { the } \varphi_{i} \text { 's are atomic formulas of }(+,-, \cdot, \wedge, \vee, \leq)\right) \\ \left|x_{j}\right| \leq a_{j} & \left(\text { all } j \in J ; \text { the } a_{j} \text { 's are in } A_{+}\right)\end{cases}
$$


If this system is finitely solvable, then it is solvable.

Proof. We first conclude in the linearly ordered case:

Claim. If $A$ is linearly ordered, then the conclusion of the theorem holds.

Proof of claim. Since $A$ is a complete linearly ordered ring, $A$ is isomorphic to $\{0\}, \mathbb{R}$ or $\mathbb{Z}$ equipped with the usual addition and the multiplication $*$ defined by $x * y=c \cdot x \cdot y$ for some positive $c$. Therefore, the operations $+,-, \cdot, \wedge, \vee$ are continuous for the interval topology of $A$ and $\leq$ is closed. We conclude easily by compactness of the topological product $\prod_{j \in J}\left[-a_{j},+a_{j}\right]$.

- Claim .

Now we prove the theorem in the general case. Without loss of generality, $A, I$ and $J$ are mutually disjoint. Furthermore, by chapter 1 , we can assume without loss of generality that all the elements of $A \cup I \cup J$ are atoms of the universe $\mathbf{W}$. Let $B$ be the complete Boolean algebra of polar subsets of $A$, and equip $A$ with the forcing defined in (4.1) and (4.2); extend $\llbracket \rrbracket$ by making $\mathbf{A} \backslash A \quad B$-scattered, and $\llbracket x=y \rrbracket=0$ for all $x$ in $A, y$ in $\mathbf{A} \backslash A$.

Now, for every finite $I^{\prime} \subseteq I$, there exists $f=\left(x_{j}\right)_{j \in J}$ in ${ }^{J} A$ such that

$$
\left(\forall i \in I^{\prime}\right)\left(A \models \varphi_{i}(f)\right) \text { and }(\forall j \in J)\left(A \models\left|x_{j}\right| \leq a_{j}\right) .
$$

Put $g=\left(a_{j}\right)_{j \in J}$. By proposition 4.3, we have

$$
\| \check{f}: \check{J} \rightarrow \check{A} \text { and }\left(\forall i \in \check{I}^{\prime}\right)\left(\check{A} \models \check{\varphi}_{i}(\check{f})\right) \text { and }(\forall j \in \check{J})(\check{A} \models|\check{f}(j)| \leq \check{g}(j)) \|=1 \text {. }
$$

This holds for all finite $I^{\prime} \subseteq I$. Using corollary 2.7, it follows easily that

$$
\begin{aligned}
\|\left(\forall \text { finite } I^{\prime} \subseteq \check{I}\right)(\exists \boldsymbol{f})\left[\boldsymbol{f}: \check{J} \rightarrow \check{A} \text { and }\left(\forall i \in I^{\prime}\right)\left(\check{A}=\check{\varphi}_{i}(\boldsymbol{f})\right)\right. \\
\quad \text { and }(\forall j \in \check{J})(\check{A} \models|\boldsymbol{f}(j)| \leq \check{g}(j))] \|=1 .
\end{aligned}
$$

By lemma 5.4 and the claim, it follows from (5.4) that

$$
\|(\exists \boldsymbol{f})\left[\boldsymbol{f}: \check{J} \rightarrow \check{A} \text { and }(\forall i \in \check{I})\left(\check{A} \models \check{\varphi}_{i}(\boldsymbol{f})\right) \text { and }(\forall j \in \check{J})(\check{A} \models|\boldsymbol{f}(j)| \leq \check{g}(j))\right] \|=1 \text {. }
$$

(we have used here the non-trivial fact - lemma $2.2-$ that $\mathbf{W}^{B}$ is a $B$-valued model of $Z F A+A C)$.

Thus, by lemma 2.3 (fullness of $\mathbf{W}^{B} \backslash \mathbf{A}$ ), there exists $\boldsymbol{f}$ in $\mathbf{W}^{B}$ such that

$$
\| \boldsymbol{f}: \check{J} \rightarrow \check{A} \text { and }(\forall i \in \check{I})\left(\check{A} \models \check{\varphi}_{i}(\boldsymbol{f})\right) \text { and }(\forall j \in \check{J})(\check{A} \models|\boldsymbol{f}(j)| \leq \check{g}(j)) \|=1 \text {. }
$$

But for all $j$ in $J$, we have $\|\boldsymbol{f}(\check{\jmath}) \in \check{A}\|=1$ and $\left\||\boldsymbol{f}(\check{\jmath})| \leq a_{j}\right\|=1$. Since $A$ is locally full (lemma 5.2), there exists $x_{j}$ in $A$ such that $\left\|\boldsymbol{f}(\check{\jmath})=x_{j}\right\|=1$; thus $\left|x_{j}\right| \leq a_{j}$. Let $f=\left(x_{j}\right)_{j \in J}$. It follows that $\|\boldsymbol{f}=\check{f}\|=1$. Thus $\left\|\check{A}=\check{\varphi}_{i}(\check{f})\right\|=1$ for all $i \in I$; again by proposition 4.3, $A \models \varphi_{i}(f)$, whence $f$ is a solution of (5.3). 
Now consider an arbitrary commutative $\ell$-group $A$; adjoin a positive infinite element $\infty$, such that $(\forall x \in A)(x<\infty)$ and $\left(\forall x \in A_{+} \cup\{\infty\}\right)(x+\infty=\infty+x=\infty)$. Note that $A$ itself cannot be algebraically compact (except when $A=\{0\}$ ), but we have the following result:

5.6. Corollary. Let $A$ be a complete -group. Then $\left(A_{+} \cup\{\infty\},+, \vee, \leq\right)$ is algebraically compact.

Proof. Put $A^{\prime}=A_{+} \cup\{\infty\}$. Let $\Gamma$ be a $(+, \vee, \leq)$-system with parameters from $A^{\prime}$, with unknowns $x_{j}(j \in J)$. Let $P$ be the set of all finite subsets of $\Gamma$, and let $\mathrm{F}$ be the filter on $P$ generated by the $F_{p}=\{q \in P: p \subseteq q\}, p \in P$. Let ${ }^{*} A^{\prime}$ be the reduced power of $A^{\prime}$ by F. For all $p$ in $P, p$ admits a solution, say $\vec{x}_{p}=\left(x_{p j}\right)_{j \in J}$. For all $j$ in $J$, let $x_{j}=\left[x_{p j}: p \in P\right] \in{ }^{*} A^{\prime}$. Then $\vec{x}=\left(x_{j}\right)_{j \in J}$ is a solution of $\Gamma$ in ${ }^{*} A^{\prime}$.

Thus to conclude, it is sufficient to prove that $A^{\prime}$ is a retract of ${ }^{*} A^{\prime}$.

Let $\mathcal{A}=\left\{\left[x_{p}\right]_{p} \in{ }^{*} A^{\prime}:\left(\exists x \in A_{+}\right)\left(\left\{p \in P: x_{p} \leq x\right\} \in \mathcal{F}\right)\right\}$. Then $A_{+}$can be identified with its image in $\mathrm{A}$ by the natural map from $A^{\prime}$ into ${ }^{*} A^{\prime}$. In fact, we have the

Claim. $A_{+}$is a retract of $A$.

Proof of claim. Let $K=\left\{(\xi, a) \in \mathcal{A} \times A_{+}: \xi \leq a\right\}$ index a family $\left(t_{\nu}\right)_{\nu \in K}$ of unknowns. Consider the following system:

$$
\begin{cases}t_{(a, b)}=a & \left(\text { all } a \leq b \text { in } A_{+}\right) \\ t_{(\xi, a)}=t_{(\xi, b)} & \text { (all } \xi, a, b \text { such that }(\xi, a) \text { and }(\xi, b) \text { are in } K) \\ t_{(\xi+\eta, a+b)}=t_{(\xi, a)}+t_{(\eta, b)} & (\text { all }(\xi, a),(\eta, b) \text { in } K) \\ 0 \leq t_{(\xi, a)} \leq a & (\text { all }(\xi, a) \in K)\end{cases}
$$

Then every finite subsystem of (5.5) is solvable in $A_{+}$(by taking $t_{(\xi, a)}$ to be some component of some representative of $\xi$ relatively to some index in a small enough set in F). Since the last group of inequalities in (5.5) gives bounds for the solutions, theorem 5.5 implies that (5.5) admits a solution, say $\left(\alpha_{(\xi, a)}\right)_{(\xi, a) \in K}$. Clearly, $\alpha_{(\xi, a)}$ depends only on $\xi$, and $\xi \mapsto \alpha_{(\xi, a)}$ defines a retract from $\mathrm{A}$ to $A_{+}$.

- Claim .

Now, let $\rho: \mathcal{A} \rightarrow A_{+}$be a retract. Then it is easy to verify that the map

$$
\bar{\rho}:{ }^{*} A^{\prime} \rightarrow A^{\prime}, \begin{cases}x \mapsto \rho(x) & \text { if } x \in \mathcal{A}, \\ x \mapsto \infty & \text { otherwise }\end{cases}
$$

is a retract from ${ }^{*} A^{\prime}$ to $A^{\prime}$. The conclusion follows.

This yields a positive answer to the question asked in [15], whether if $A$ is a complete

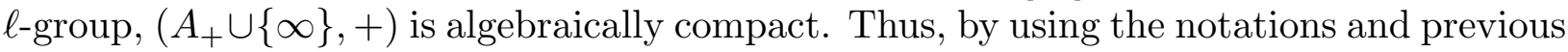
results of [15] (recall that 2-CHEP stands for "2-charge extension property"), we obtain the following

5.7. Corollary. Let $A$ be a partially ordered group. Then the following are equivalent:

(i) $A_{+}$has the 2-CHEP;

(ii) $A_{+} \cup\{\infty\}$ has the 2-CHEP;

(iii) $A$ is a complete $\ell$-group. 
For more general structures than $A_{+} \cup\{\infty\}$, we do not have a complete answer. The proper context for this question is the following; define, as in [15], [17], a P.O.M. to be a commutative monoid $A$ equipped with a partial preordering $\leq$ satisfying $(\forall x)(x \geq 0)$ and $(\forall x, y, z)(x \leq y \Rightarrow x+z \leq y+z)$. A P.O.M. is minimal (resp. antisymmetric) when it satisfies $(\forall x, y)(x \leq y \Leftrightarrow(\exists z)(x+z=y))$ (resp. $(\forall x, y)((x \leq y$ and $y \leq x) \Rightarrow x=y))$. A class of P.O.M.'s that will be of importance to us is the class of complete P.O.M.'s. By definition, a complete P.O.M. is a minimal, antisymmetric P.O.M. $E$ satisfying the following conditions (recall - see $[17]$ - that $x \ll y$ stands for $x+y=y$ ):

$$
\begin{gathered}
(\forall X \subseteq E)(\bigwedge X \text { exists }) ; \\
(\forall a \in E)(\forall X \subseteq E)(\bigwedge(a+X)=a+\bigwedge X) ; \\
(\forall a, b, c)(a+c \leq b+c \Rightarrow(\exists d \ll c)(a \leq b+d)) ; \\
(\forall a \in E)(\forall X \subseteq E)(X \ll a \Rightarrow \bigvee X \ll a) .
\end{gathered}
$$

In particular, if $A$ is a complete $\ell$-group, then $A_{+} \cup\{\infty\}$ is a complete P.O.M.. Complete P.O.M.'s seem very short of being algebraically compact: indeed, it is proven in [17] that injective P.O.M.'s are exactly complete and $\mathbb{N}$-divisible P.O.M.'s; in addition, all the relevant finite and infinite identities satisfied by injective P.O.M.'s are already satisfied by complete P.O.M.'s. And furthermore, completeness is the least we have to assume to ensure algebraic compactness, as the following proposition shows:

Proposition 5.8. Let $E$ be a minimal, antisymmetric P.O.M. satisfying the following finite refinement property:

$\left(\forall a_{0}, a_{1}, b_{0}, b_{1}\right)\left(a_{0}+a_{1}=b_{0}+b_{1} \Rightarrow\left(\exists_{i, j<2} c_{i j}\right)\left(\bigwedge_{i<2}\left(a_{i}=c_{i 0}+c_{i 1}\right)\right.\right.$ and $\left.\left.\bigwedge_{j<2}\left(b_{j}=c_{0 j}+c_{1 j}\right)\right)\right)$

If $(E,+)$ is algebraically compact, then $E$ is a complete P.O.M..

Proof. First, $(E,+, \leq)$ is also algebraically compact (because in any atomic system, every inequality $s \leq t$, where $s$ and $t$ are terms, can be replaced by the equation $s+x=t$ where $x$ is a new unknown). Now, we prove that $E$ is a complete P.O.M., in a series of claims. We refer the reader to [17], from which we use both terminology and results.

Claim 1. Let $A \subseteq E$ be upwards directed. Then $\bigvee A$ exists.

Proof of claim. Let $S=\{x \in E: A \leq x\}$. Consider the following atomic system, with unknown $x$ :

$$
\begin{cases}a \leq x & (\text { all } a \in A) \\ x \leq s & (\text { all } s \in S)\end{cases}
$$

Since $A$ is upwards directed, (5.6) is finitely solvable, thus solvable by hypothesis. If $x$ is a solution of (5.6), then $x=\bigvee A$.

- Claim 1. 
Thus, for all $a$ in $E, \infty a=\bigvee\{n a: n \in \mathbb{N}\}$ is well-defined.

Claim 2. Let $A \subseteq E$ be a upwards directed, nonempty subset of $E$, and let $a \in E$. Then $\bigvee(a+A)=a+\bigvee A$.

Proof of claim. Put $b=\bigvee(a+A), c=\bigvee A$. Obviously, $b \leq a+c$. Conversely, consider the following atomic system, with unknown $x$ :

$$
\left\{\begin{array}{l}
u \leq x \\
a+x \leq b
\end{array} \quad(\text { all } u \in A)\right.
$$

Then (5.7) is finitely solvable (because $A$ is upwards directed, and $A \neq \emptyset$, thus $a \leq b$ ), thus solvable by hypothesis. Let $x$ be a solution of (5.7). Then $a+c \leq a+x \leq b$. Thus $b=a+c$.

- Claim 2.

Claim 3. $(\forall a \in E)(a+\infty a=\infty a=2(\infty a))$.

Proof of claim. A simple calculation, using claim 2:

$$
\begin{aligned}
a+\infty a & =a+\bigvee\{n a: n \in \mathbb{N}\} \\
& =\bigvee\{(n+1) a: n \in \mathbb{N}\} \\
& =\infty a,
\end{aligned}
$$

and

$$
\begin{aligned}
\infty a+\infty a & =\infty a+\bigvee\{n a: n \in \mathbb{N}\} \\
& =\bigvee\{\infty a+n a: n \in \mathbb{N}\} \\
& =\infty a
\end{aligned}
$$

- Claim 3. $1.2])$

Claim 4. E is a strong refinement P.O.M.. (see [17, definition 1.12] or [15, definition

Proof of claim 4. Let $a, b, c$ in $E$ such that $a+c \leq b+c$. Consider the following atomic system, with unknown $x$ :

$$
\left\{\begin{array}{l}
a \leq b+x \\
n x \leq b
\end{array} \quad(\text { all } n \in \mathbb{N})\right.
$$

Since $E$ satisfies the finite refinement property and by [17, lemma 1.11], (5.8) is finitely solvable, thus solvable. Let $d$ be a solution of (5.8). Then $\infty d \leq b$, thus $d \ll b$ by claim 3 , and $a \leq b+d$.

- Claim 4. 
Claim 5. For every subset $A$ of $E, \wedge A$ exists.

Proof of claim. Let $S=\{s \in E: s \leq A\}$. Consider the following atomic system, with unknown $x$ :

$$
\begin{cases}s \leq x & (\text { all } s \in S) \\ x \leq a & (\text { all } a \in A)\end{cases}
$$

Since $E$ is a strong refinement P.O.M., it satisfies the finite interpolation property ([17, lemma $1.16,(\mathrm{i})])$. Thus, (5.9) is finitely solvable. Let $x$ be a solution of (5.9). Then $x=\bigwedge A$.

- Claim 5.

Claim 6. For all $a \in E$ and $A \subseteq E, \bigwedge(a+A)=a+\bigwedge A$.

Proof of claim. Put $b=\bigwedge A, c=\bigwedge(a+A)$. It is trivial that $a+b \leq c$. Conversely, consider the following atomic system, with unknown $x$ :

$$
\left\{\begin{array}{l}
c \leq a+x \\
x \leq u
\end{array} \quad(\text { all } u \in A)\right.
$$

Then (5.10) is finitely solvable (use [17, lemma 1.16,(iv)]), thus solvable. If $x$ is a solution of (5.10), then $c \leq a+x \leq a+b$.

- Claim 6.

Claim 7. Let $a \in E, X \subseteq E$ such that $X \ll a$. Then $\bigvee X \ll a$.

Proof of claim. Consider the following atomic system, with unknown $x$ :

$$
\left\{\begin{array}{l}
x+a=a \\
u \leq x
\end{array} \quad(\text { all } u \in A)\right.
$$

Then (5.11) is finitely solvable, thus solvable. Let $x$ be a solution of (5.11). Then $\bigvee X \leq$ $x \ll a$ (and $E$ is antisymmetric), thus $\bigvee X \ll a$.

- Claim 7.

Thus, the proof is complete.

We do not know whether the converse of proposition 5.8 holds. However, the methods developed in this paper allow us to prove the following partial converse:

5.9. Theorem. Let $E$ be a complete P.O.M. satisfying the following additional conditions:

(i) For each $p$ in $E, \perp p$ admits a largest element, which we will denote by $p^{\perp}$;

(ii) $(\forall p)\left(p^{\perp}+p^{\perp \perp}=\infty\right)$;

(iii) $(\forall a, b)((a \backslash b) \wedge(b \backslash a)=0)$ (Where, as in [17], $a \backslash b$ is the largest $x$ in $E$ such that $a \leq b+x)$.

Then $(E,+, \vee, \wedge, \leq)$ is algebraically compact.

Proof. We start with the linearly ordered case: 
Claim 1. Let E be a linearly ordered complete P.O.M.. Then E, equipped with its interval topology, is compact Hausdorff, and $+, \vee, \wedge$ are continuous; thus $E$ is algebraically compact.

Proof of claim. Since $(E, \leq)$ is a complete chain, the interval topology on $E$ is compact Hausdorff (see [4]). Now, since $(E, \leq)$ is a complete chain, the interval topology and the order topology on $E$ are the same [4], thus, to verify that the addition is continuous, it suffices to verify that if $a, b \in E$ and $\mathrm{D}$ is a directed set and $\left(x_{\nu}\right)_{\nu \in \mathcal{D}},\left(y_{\nu}\right)_{\nu \in \mathcal{D}}$ are two convergent nets with respective limits $a$ and $b$, the net $\left(x_{\nu}+y_{\nu}\right)_{\nu \in \mathcal{D}}$ converges to $a+b$. Since $E$ is a complete chain, it suffices to consider the four cases where $\left(x_{\nu}\right)_{\nu \in \mathcal{D}}$ (resp. $\left(y_{\nu}\right)_{\nu \in \mathcal{D}}$ ) is increasing (resp. decreasing) with limit $a$ (resp. b). Then, the distributivity of + on $\bigwedge$ and on $\bigvee \neq \emptyset([17$, proposition 2.14]) allows us to conclude easily. $\quad$ Claim 1.

Now we prove the theorem in the general case. So consider a finitely solvable atomic system with parameters form $E$ :

$$
\left\{\varphi_{i}(\vec{x}) \quad(\text { all } i \in I) \text { with } \vec{x}=\left(x_{j}\right)_{j \in J} .\right.
$$

As usual, we can assume without loss of generality that $E, I$ and $J$ are mutually disjoint and that all elements of $A \cup I \cup J$ are atoms of the universe $\mathbf{W}$. As in the case of $\ell$-groups, for all $p, q$ in $E$, we put $p \mid q \Leftrightarrow\left(\exists^{\neq 0} r\right)(r \leq p, q)$, then $p \perp q \Leftrightarrow \neg(p \mid q)$, then $p \| q \Leftrightarrow\left(\forall^{\neq 0} r \leq p\right)(r \mid q)$. Furthermore, we can equip $E$ with the following definition of forcing: for all $p, a, b$ in $E$,

$$
\left\{\begin{array}{l}
p \Vdash a \leq b \Longleftrightarrow p \perp a \backslash b, \\
p \Vdash a=b \Longleftrightarrow(p \Vdash a \leq b \text { and } p \Vdash b \leq a) .
\end{array}\right.
$$

It turns out that in the context of the hypotheses (i), (ii), (iii) above, this definition is equivalent to the general one of [13], but we will not be concerned about this here.

Then, let $B$ be the completion (see [11]) of the partially ordered set $E \backslash\{0\}$, let $p \mapsto[p]$ be the natural map from $E \backslash\{0\}$ to $B$ (and put $[0]=0_{B}$ ). Define Boolean values as in (4.3). Then, we have the following

Claim 2. $E$ is a B-valued model, for the language $(+, \leq)$, of the theory of linearly ordered antisymmetric, minimal P.O.M.'s. Furthermore, for all $a$, $b$ in $E,\|a \leq b\|=1$ (resp. $\|a=b\|=1$ ) if and only if $a \leq b$ (resp. $a=b)$.

Proof of claim. It suffices to check that for all $p$ in $E \backslash\{0\}$ and all $a, a^{\prime}, b, b^{\prime}, c$ in $A$, the following holds:

$$
\left\{\begin{array}{l}
p \Vdash 0 \leq a ; \\
p \Vdash a \leq a ; \quad(p \Vdash a \leq b \text { and } p \Vdash b \leq a) \Rightarrow p \Vdash a=b ; \\
(p \Vdash a \leq b \text { and } p \Vdash b \leq c) \Rightarrow p \Vdash a \leq c ; \\
p \Vdash a=a ; \quad p \Vdash a=b \Leftrightarrow p \Vdash b=a ; \quad(p \Vdash a=b \text { and } p \Vdash b=c) \Rightarrow p \Vdash a=c ; \\
\left(p \Vdash a \leq b \text { and } p \Vdash a=a^{\prime} \text { and } p \Vdash b=b^{\prime}\right) \Rightarrow p \Vdash a^{\prime} \leq b^{\prime} ; \\
\left(p \Vdash a \leq a^{\prime} \text { and } p \Vdash b \leq b^{\prime}\right) \Rightarrow p \Vdash a+b \leq a^{\prime}+b^{\prime} ; \\
\left(p \Vdash a=a^{\prime} \text { and } p \Vdash b=b^{\prime}\right) \Rightarrow p \Vdash a+b=a^{\prime}+b^{\prime} ; \\
\text { If } p \Vdash a \leq b \text { and } c=b \backslash a, \text { then } p \Vdash a+c=b . \\
\left(\forall^{*} p\right)(p \Vdash a \leq b \text { or } p \Vdash b \leq a) .
\end{array}\right.
$$


The proof of all these facts with the exception of the last one is straightforward, using easily established facts as $a \backslash a=0, a \backslash c \leq(a \backslash b)+(b \backslash c),(a+b) \backslash\left(a^{\prime}+b^{\prime}\right) \leq\left(a \backslash a^{\prime}\right)+\left(b \backslash b^{\prime}\right)$, $(a+(b \backslash a)) \backslash b=(a \vee b) \backslash b=a \backslash b$. For the last of these facts, if $\left(\exists^{\neq 0} q \leq p\right)(q \Vdash a \leq b)$ then we are done; otherwise, we have $(\forall \neq 0 q \leq p)(q \| a \leq b)$, thus $p \Vdash a \backslash b$; thus $p \perp \bar{b} \backslash a$ by the hypothesis (iii), i.e. $p \Vdash b \leq a$. The conclusion of the first part follows. For the second part, it suffices to give the proof for $\leq$. It is obvious that $a \leq b$ implies $\|a \leq b\|=1$. Conversely, suppose that $\|a \leq b\|=1$. If $a \not \leq b$, then there exists $p \leq a \backslash b$ non zero such that $p \Vdash a \leq b$, whence $p \perp a \backslash b$. Thus $p=0$, a contradiction. Thus $a \leq b$.

- Claim 2.

This claim allows us, once again, to define inductively the Boolean value of any formula of $(+, \leq)$ in a truth-preserving way. Furthermore, we extend the function $\llbracket-\rrbracket$ to the whole $\mathbf{W}^{B}$ as in (2.2). To go on, the analogue of the claim of proposition 4.3 is not as easy here, because \is not as wieldy as - in groups. However, the following claim provides us a way out of this slight difficulty:

Claim 3. Let $X$ be a subset of $E$, let $a$ in $E$. Then $a \backslash \bigwedge X=\bigvee(a \backslash X)$ and $\bigvee X \backslash a=\bigvee(X \backslash a)$.

Proof of claim. Put $b=\bigwedge X, c=\bigvee(a \backslash X)$. Then $c \leq a \backslash b$ is trivial. Conversely, for all $x$ in $X$, we have $a \backslash x \leq c$, i.e. $a \leq c+x$, i.e. $a \backslash c \leq x$; thus $a \backslash c \leq b$, whence, as before, $a \backslash b \leq c$. Thus $a \backslash b=c$.

Now, put $b^{\prime}=\bigvee X, c^{\prime}=\bigvee(X \backslash a)$. Thus $c^{\prime} \leq b^{\prime} \backslash a$ is trivial. Conversely, for all $x$ in $X, x \backslash a \leq c^{\prime}$, i.e. $x \leq a+c^{\prime}$; thus $b^{\prime} \leq a+c^{\prime}$, whence $b^{\prime} \backslash a \leq c^{\prime}$. Thus $b^{\prime} \backslash a=c^{\prime}$.

- Claim 3.

This allows us to prove the following claim, similar to proposition 4.5:

Claim 4. Let $a \in E, X \subseteq E$. Then

(i) $a=\bigwedge X$ if and only if $\|a=\bigwedge \check{X}\|=1$;

(ii) $a=\bigvee X$ if and only if $\|a=\bigvee \check{X}\|=1$.

Proof of claim. Similar to the proof of proposition 4.5, using claim 3 and the hypothesis (i).

- Claim 4.

In particular, when $X$ is a pair, we find the analogue of the claim of proposition 4.3.

Claim 5. E is a projectable B-valued model.

Proof of claim. Let $u$ in $B, x, y$ in $E$. Then $a=\bigvee_{p \in u} p^{\perp \perp}$ and $b=\bigwedge_{p \in u} p^{\perp}$ are mutually disjoint by the hypothesis (i), and $a+b=\infty$ by distributivity of the addition on $\bigwedge$ and on $\bigvee \neq \emptyset$. Furthermore, $b \in \perp u$ by definition; finally, we prove that $a \in u$, using the fact that $u$ is regular-open, i.e. we prove that $\left(\forall^{\neq 0} p \leq a\right)\left(\exists^{\neq 0} q \leq p\right)(q \in u)$. So let $p \neq 0, p \leq a$. By the hypothesis (i), there exists $r$ in $u$ such that $p \wedge r^{\perp \perp} \neq 0$. Thus, $p \wedge r \neq 0$; but $u$ is open, thus $p \wedge r \in u$, which proves the fact: thus $a \in u$.

Thus, we have proved that $a \in u, b \in \perp u$ and $a+b=\infty$. Let $z=x \wedge a+y \wedge b$. Then it is immediate that $u \leq\|z=x\|$ and $\neg u \leq\|z=y\|$.

- Claim 5.

We can now prove the

Claim 6. $\| \check{E}$ is a linearly ordered complete P.O.M. $\|=1$. 
Proof of claim. Let $p, a, b, c$ in $E$ such that $p \Vdash a+c \leq b+c$. Let $d=c-c=$ largest $x$ such that $x \ll c($ see $[17$, definition 2.21]). Put $x=(a+c) \backslash(b+c)$. Then $a+c \leq b+c+x$, thus $a \leq b+d+x$, i.e. $a \backslash(b+d) \leq x$; but $p \perp x$, whence $p \Vdash a \leq b+d$. Thus, we have proved that

$$
\|(\forall a, b, c)(a+c \leq b+c \Rightarrow(\exists d \ll c)(a \leq b+d))\|=1 .
$$

Since $E$ is projectable (claim 5), it remains, by lemma 2.10, to show that for all $X \subseteq E, \| \bigwedge \check{X}$ exists $\|=1$ and for all $a$ in $E,\|a+\bigwedge \check{X}=\bigwedge(a+\check{X})\|=1$ and $\| \check{X} \ll a \Rightarrow$ $\bigvee \check{X} \ll a \|=1$. However, this is an easy application of claims 3 and 4 . We conclude by claim 2 .

- Claim 6.

Claim 7. E is full.

Proof of claim. It suffices to prove that for every antichain $W$ of $E \backslash\{0\}$ and for every family $\left(x_{p}\right)_{p \in W}$ of elements of $E$, there exists $x$ in $E$ such that $(\forall p \in W)\left(p \Vdash x=x_{p}\right)$. Using both hypotheses (i) and (ii), it is clear that $x=\bigvee_{p \in W}\left(x_{p} \wedge p^{\perp \perp}\right)$ satisfies the given property.

- Claim 7.

Now, we can conclude as at the end of the proof of theorem 5.5, the argument being made slightly simpler by the fact that $E$ is full and not just locally full.

5.10. Corollary. Under the hypotheses of theorem 5.9, E satisfies the following infinite distributive law:

$$
(\forall a \in E)(\forall X \subseteq E)(a \wedge \bigvee X=\bigvee(a \wedge X))
$$

Proof. Put $b=\bigvee X$ and $c=\bigvee(a \wedge X)$. It is obvious that $c \leq a \wedge b$. Conversely, consider the following atomic system, with unknown $x$ :

$$
\left\{\begin{array}{l}
a \wedge x \leq c \\
s \leq x
\end{array} \quad(\text { all } s \in X)\right.
$$

By [17, lemma 1.16, (vii)], (5.15) is finitely solvable. By theorem 5.9, (5.15) is solvable; let $x$ be a solution of (5.15), then we have $b \leq x$ and $a \wedge x \leq c$, whence $a \wedge b \leq c$; thus $a \wedge b=c$.

The proof of corollary 5.10 also shows that not all complete P.O.M.'s are algebraically compact in the language $(\wedge)$ : this is because they would then satisfy $(5.14)$, which is not always the case, for example for $\mathbb{N}^{2} \cup\{\infty\}$. Note that the dual property of (5.14) is always true (see [17, proposition 2.19]).

Note also that if $E$ is an idem-multiple complete P.O.M., then $E$ is an injective P.O.M. by [17, theorem 3.11$]$, thus $(E,+, \leq)$ is a fortiori algebraically compact (because it is a retract of its reduced powers, see e.g. [16]). But this hypothesis seems rather to stand on the opposite side as the one of theorem 5.9, with the same conclusion. This leads us to the following 
5.11. Question. Let $E$ be a complete P.O.M.. Is $(E,+, \leq)$ algebraically compact? (Or, equivalently, is $(E,+)$ equationally compact?)

A related question is the following:

5.12. Question. Let $E$ be a weak cardinal algebra [17, definition 2.2]. Is $(E,+, \leq)$ "countably algebraically compact", i.e. every countable atomic finitely solvable system with parameters from $E$ is solvable in $E$ ?

Again, a strong evidence for an affirmative answer to this question is provided by [18, corollary 4.5], which states that every divisible weak cardinal algebra is "countably injective" (thus in particular countably algebraically compact).

\section{$\S 6$. Afterword: generalization to naive set theory.}

We shall indicate here informally how all the proofs of "non set-theoretical" results of this paper can be naturally translated into naive set theory (for example Zermelo's set theory $Z$, or $Z C=Z+A C$, or versions with atoms $Z A$, etc.), but also with potential extension to other set theories. Although a lot of it may be already known to set theorists (which explains our choice of keeping informal), we do not know of any publication where it is written. At any rate, it is here that the importance of choosing to construct our models with atoms rather than to embed Boolean models into the Scott-Solovay universe (as in [13]) will appear fully. Unfortunately, it does not seem to be possible to avoid many more or less cumbersome details. The probable reason for that must be that while Zermelo's set theory $Z$ (or $Z C$ ) is well-adapted to what could be called "classical" mathematical practice, it is on the contrary rather unwieldy when it comes to set-theoretical constructions; on the contrary, there are set theories that are intuitively much weaker than $Z$ when it comes to classical mathematical practice (e.g. admissible set theory $K P$, or $K P U$ if we want atoms - see [1]), but much better adapted to set-theoretical constructions. We shall now draw the lines of a "naive set theory" that still allows set-theoretical constructions as e.g. the ones used in this paper.

A first thing to do is to modify some of the axioms displayed in $\S 1$ to adapt them to the context of naive set theory. First, the collection scheme is simply to be suppressed. Next, the axiom of infinity has to be changed into a weaker form (equivalent under $Z F$ ), the point being that the class $\omega$ of all finite ordinals may no longer be an object of the universe. For example, we can take the axiom "there is an infinite set", finiteness still being understood as Tarski-finiteness, or, equivalently, equipotence with a finite ordinal: if $X$ is an infinite set, let $\Omega$ be the set of all finite subsets of $X$, and define the set $\mathbb{N}$ of natural integers to be the quotient of $\Omega$ by the equipotence relation.

Finally, the axiom of regularity has to be changed in a more radical way, guaranteeing that every set has finite rank (there is no contradiction with the axiom of infinity stated above, because $\omega$ may not be a set). For example, it can be stated as

$$
\begin{aligned}
& (\forall x)(\exists n \in \mathbb{N}) \neg(\exists f)(f \text { is a function of domain }[0, n] \\
& \text { and } f(0)=x \text { and }(\forall i<n)(f(i+1) \in f(i)))
\end{aligned}
$$


Then, denote by $Z A$ the new axiom system.

Now, let $A$ be any set in a universe $\mathfrak{U}$ of, say, $Z$. The construction of $\mathbf{V}_{A}$ as presented in $\S 1$ changes accordingly: namely, (1.1) is carried over for finite $\xi$, and then $\mathbf{V}_{A}=$ $\bigcup_{n \in \mathbb{N}} \mathbf{V}_{A, n}$. Then $\mathbf{V}_{A}$ satisfies all the axioms of $Z A$, with the possible exceptions of the (new) axiom of infinity (if $A$ is finite!) and choice (the basic $\mathfrak{U}$ may not itself satisfy $A C$ !). But of course, if $A$ is infinite, then $\mathbf{V}_{A}$ satisfies the axiom of infinity and if $\mathfrak{U}$ satisfies the axiom of choice (or even if all the $\mathcal{P}^{n}(A)$ 's are well-orderable in $\mathfrak{U}$ ) then $\mathbf{V}_{A}$ satisfies the axiom of choice; to prove this, one can use an analogue of proposition 1.2 (ii) (for finite $\alpha)$.

Actually, in order to be able to generalize $\S 2$, we will need slightly more than the fact that $\mathbf{V}_{A}$ satisfies $A C$. A proper way to proceed is the following: let $S$ be another set of $\mathfrak{U}$, of the form $\mathbb{N} \times S^{\prime}$ (so that $S$ is canonically equipotent to $\mathbb{N} \times S$ ), infinite, disjoint from $A$, such that there exists a one-to-one map from $A$ to $S$ (which we will abbreviate by $A \hookrightarrow S$ ). Let $A^{\prime}=A \cup S$, and consider the universe $\mathbf{V}_{A^{\prime}}$ constructed with the modification indicated above. Then $\mathbf{V}_{A^{\prime}}$ satisfies the axiom of infinity, and if $\mathfrak{U}$ satisfies $A C$, then $\mathbf{V}_{A^{\prime}}$ satisfies $A C$. Furthermore, it is not difficult to prove that in fact, $\mathbf{V}_{A^{\prime}}$ satisfies the statement

$$
(\forall \text { set } x)(\exists n \in \mathbb{N})\left(x \hookrightarrow \mathcal{P}^{n}(S)\right)
$$

Now, we must show how to change the constructions of $\S 2$. So we start within $\mathbf{W}=$ $\mathbf{V}_{A^{\prime}}$; once again, the construction (2.1) is performed only at finite level. Here, $A$ is already given a structure of $B$-valued model of the equality. Then, the map [- $\rrbracket$ is extended to the whole $A^{\prime}=A \cup S$ by making $S B$-scattered and $B$-separated from $A$, i.e. $\|x=y\|=0$ whenever $x \neq y$ and either $x, y \in S$ or $x \in A$ or $y \in S$, or $y \in A$ and $x \in S$. Then, the definition (2.2) is still coherent (since all ranks are finite), and the proof of lemma 2.1 does not bring any trouble. The situation for lemma 2.2 is different: all axioms of $Z A$ are easily proved to hold in $\mathbf{W}^{B}$, except the axiom of infinity and, if $\mathbf{W}$ satisfies $A C$, the axiom of choice. But in fact, the axiom of infinity is easily dealt with because $S$ is infinite and $B$-scattered, so it remains to prove the axiom of choice (in case $\mathbf{W}$ satisfies it). The point is that to be a well-ordering is no longer uniform (definition 2.4): this is because being antisymmetric is not a uniform formula, as easy examples show. On the other hand, to be a well-ordering on a given $B$-scattered set is $B$-uniform, with a similar argument to the one in the proof of the axiom of regularity in $\mathbf{V}^{B}$ shown in [11, theorem 43]. And the importance of choosing carefully $S$ as above appears here fully: in $\mathbf{W}^{B}$, every nonempty set is the surjective image of some set in $\mathbf{W}$ (by using the interpretation function [11, chapter 18] in the classical way), thus of some $\mathcal{P}^{n}(S)$ in $\mathbf{W}$; but these are $B$-scattered and can be well-ordered, thus, in $\mathbf{W}^{B}$, every set can be well-ordered. Thus, $\mathbf{W}^{B}$ satisfies the axiom of choice.

From then on, no major problem arises. This provides us with a canonical way to prove all the results of this paper in naive set theory, without modification in the case of non set-theoretical results and with obvious modifications in the case of set-theoretical results. The same holds for the results in [13], as e.g. the fact that every Boolean-linear space admits a completion. There is probably more to say about this, using e.g. admissible set 
theory rather than naive set theory, perhaps to obtain relativizations of the results shown here. However, we have not investigated this aspect of things.

\section{BIBLIOGRAPHY.}

[1] J. BARWISE, "Admissible sets and structures", Springer-Verlag, 1975.

[2] S.J. BERNAU, "Lateral and Dedekind-completion of Archimedean lattice groups", Journal of the London Mathematical Society, Vol. 12, part 3, pp. 320-322, 1976.

[3] A. BIGARD, K. KEIMEL and S. WOLFEnStEIN, "Groupes et anneaux réticulés", Lecture Notes in Mathematics, Springer-Verlag, 1977.

[4] G. BIRKHOFF, "Lattice theory", American Mathematical Society, Providence, RI, 1967.

[5] C.C. CHANG and H.J. KEISLER, "Model Theory", North Holland, 1973.

[6] L. FUCHS, "Infinite abelian groups", Academic Press, Vol. 1 (1970) and Vol. 2 (1973).

[7] G. GIERZ, K.H. HOFMANN, K. KEIMEL, J.D. LAWSON, M. MiSLOVE and D.S. SCOTT, "A compendium of continuous lattices", Springer-Verlag, 1980.

[8] J. JAKUBÍK, "On $\sigma$-complete lattice-ordered groups", Czechoslovak Mathematical Journal 23 (98), pp. 164-174, 1973.

[9] J. JAKUBÍK, "Conditionally orthogonally complete $\ell$-groups", Math. Nachr. 65, pp. 153-162, 1975.

[10] T. JECH, "The axiom of choice", North-Holland, 1973.

[11] T. JECH, "Set Theory", Academic Press, 1978.

[12] T. JECH, "Boolean-valued models", in Handbook of Boolean algebras (Edited by J.D. Monk), NorthHolland, Vol. 3, pp. 1197-1211, 1989.

[13] T. JECH, "Boolean-linear spaces", Advances in Mathematics 81, pp. 117-197, 1990.

[14] J.L. KELLEY, "General Topology", Van Nostrand, revised edition 1968.

[15] R.M. SHORTT and F. WEHRUNG, "Common extensions of semigroup-valued charges", to appear in Journal of Mathematical Analysis and Applications.

[16] B. WEGLORZ, "Equationally compact algebras (I)", Fundamenta Mathematicae 59 (1966), pp. 289298 (1966).

[17] F. WEHRUNG, "Injective positively ordered monoids I", Journal of Pure and Applied Algebra 83 (1992), pp. 43-82.

[18] F. WEHRUNG, "Restricted injectivity, transfer property and decompositions of separative positively ordered monoids", to appear in Communications in Algebra. 